\title{
Spectral geometry and asymptotically conic convergence
}

\author{
Julie Rowlett
}

We define a new conic metric collapse, asymptotically conic convergence, ${ }^{1}$ in which a family of smooth Riemannian metrics degenerates to have an isolated conic singularity. For a conic metric $\left(M_{0}, g_{0}\right)$ and an asymptotically conic or "scattering" metric $\left(Z, g_{z}\right)$, we construct a new non-standard blowup, the resolution blowup, in which the conic singularity in $M_{0}$ is resolved by $Z$. This blowup induces a smooth family of metrics $\left\{g_{\epsilon}\right\}$ on the compact resolution space $M .\left(M, g_{\epsilon}\right)$ is said to converge asymptotically conically to $\left(M_{0}, g_{0}\right)$ as $\epsilon \rightarrow 0$.

Let $\Delta_{\epsilon}$ and $\Delta_{0}$ be geometric Laplacians on $\left(M, g_{\epsilon}\right)$ and $\left(M_{0}, g_{0}\right)$, respectively. Our first result is convergence of the spectrum of $\Delta_{\epsilon}$ to the spectrum of $\Delta_{0}$ as $\epsilon \rightarrow 0$. Note that this result implies spectral convergence for the $k$-form Laplacian under certain geometric hypotheses. This theorem is proven using rescaling arguments, standard elliptic techniques and the $b$-calculus of [33]. Our second result is technical: we construct a parameter $(\epsilon)$ dependent heat operator calculus which contains, and hence describes precisely, the heat kernel for $\Delta_{\epsilon}$ as $\epsilon \rightarrow 0$. The consequences of this result include the existence of a polyhomogeneous asymptotic expansion for $H_{\epsilon}$ as $\epsilon \rightarrow 0$, with uniform convergence down to $t=0$. To prove this result, we construct heat spaces as manifolds with corners using both standard and non-standard blowups on which we construct suitable heat operator calculi. A parametrix construction modeled after Melrose's heat kernel construction [33] and a maximum principle argument complete the proof.

\section{Introduction}

In the 1970s and 80s, Cheeger [6] initiated a study of the spectrum of the Laplacian and heat kernel on manifolds with isolated conical singularities. Simultaneously, Melrose [33] developed pseudodifferential techniques

\footnotetext{
${ }^{1}$ The name "asymptotically conic convergence" was chosen because the geometry of the collapsing neighborhood is described by an asymptotically conic space.
} 
to study elliptic operators on manifolds with cylindrical ends and conic singularities. Other works include $[2,3,24,34,35]$. Manifolds with isolated conical singularities are instructive models for the more singular CalabiYau orbifolds which are of particular interest to physicists, see for example [21]. Although an isolated conical singularity is the simplest example of a metric singularity, the spectral geometry of Riemannian manifolds with isolated conic singularities is still not completely understood; see, for example, $[29,31]$. One approach to understanding the spectral geometry of a singular space is to consider a family of smooth manifolds which converge in some sense to the singular space; that is the approach taken here and in $[7-9,11,13,25,26,30,32]$. The goal of our somewhat lengthy and technical spectral convergence results is to provide new tools for understanding the spectral geometry of manifolds with isolated conic singularities. Our results also apply to the singular spaces studied in [10,29]. With sufficient control on the metric degeneration, uniform spectral convergence results for $k$-form Laplacians and their heat kernels are applicable to understanding more subtle spectral invariants such as the $\eta$ invariant, as in [30]. Our work is in the spirit of [30] and generalizes the work of McDonald [32]. We briefly recall the setup and main results of those authors.

Let $M$ be a fixed compact manifold with Riemannian metric, and let $H$ be an embedded orientable hypersurface with defining function $x$ and smooth metric $g_{H}$. Let

$$
g_{\epsilon}:=d x^{2}+\left(\epsilon^{2}+x^{2}\right) g_{H}, \quad \epsilon \in[0,1)
$$

As $\epsilon \rightarrow 0, g_{\epsilon} \rightarrow d x^{2}+x^{2} g_{H}$, which has an isolated conic singularity at $x=0$. Geometrically, $M$ is pinched along the hypersurface $H$ as $\epsilon \rightarrow 0$ and the resulting metric has a conic singularity at $x=0$. The study of this metric collapse is the content of the 1990 thesis of McDonald [32]. In 1995, Mazzeo and Melrose [30] developed pseudodifferential techniques to describe the behavior of the spectral geometry under another specific type of metric collapse known as analytic surgery. As $\epsilon \rightarrow 0$, the metrics

$$
\frac{|d x|^{2}}{x^{2}+\epsilon^{2}}+h=g_{\epsilon} \longrightarrow g_{0}=\frac{|d x|^{2}}{x^{2}}+h \text {. }
$$

$g_{0}$ is an exact $b$-metric on the compact manifold with boundary $\bar{M}$ obtained by cutting $M$ along $H$ and compactifying as a manifold with boundary, hence the name, analytic surgery. Under certain assumptions on the associated 
Dirac operators, ${ }^{2}$ they proved

$$
\lim _{\epsilon \rightarrow 0} \eta\left(\partial_{\epsilon}\right)=\eta_{b}\left(\partial_{\bar{M}}\right)
$$

where $\eta_{b}\left(\partial_{\bar{M}}\right)$ is the $b$-version of the eta invariant introduced by Melrose. These results were proven by analyzing the resolvent family of the Dirac operators $\partial_{\epsilon}$ uniformly near zero. This led to a precise description of the behavior of the small eigenvalues. In our work, we obtain uniform convergence results for heat kernels under conic metric degeneration. Since many interesting spectral invariants (including the $\eta$ invariant) are computable via the heat kernel and its trace, it is reasonable to expect that our results will yield applications like [30]. Our results are necessarily quite technical; without such a detailed analysis, it is simply not possible to calculate spectral invariants, see, for example, Perelman's counterexample in $[9]$.

The metric degeneration we consider, asymptotically conic convergence (acc), encompasses the conic collapse of [32], the analogous smooth collapse of a higher codimension submanifold and the collapse of an open neighborhood with some restrictions on the local geometry. Before stating our spectral convergence results for geometric Laplacians, we recall their definition.

Definition 1.1. Let $(E, \nabla)$ be a Hermitian vector bundle over a Riemannian manifold $(M, g)$ with metric-compatible connection $\nabla$. A geometric Laplacian is an operator $\Delta$ acting on sections of $E$ which has the form

$$
\Delta=\nabla^{*} \nabla+R
$$

where $R$ is a non-negative self-adjoint endomorphism of $E$. By the Weitzenböck Theorem [36], the Laplacian on $k$-forms is a geometric Laplacian, as is the Hodge Laplacian and the conformal Laplacian; any geometric Laplace-type operator is a geometric Laplacian.

Our results are the following.

Theorem 5.1. Let $\left(M_{0}, g_{0}\right)$ be a compact Riemannian $n$-manifold with isolated conic singularity, and let $\left(Z, g_{z}\right)$ be an asymptotically conic (ac)

\footnotetext{
${ }^{2}$ In a later collaboration with Hassell et al. [18], these hypotheses were removed.
} 
space, with $n \geq 3$. Assume $\left(M, g_{\epsilon}\right)$ converges asymptotically conically to $\left(M_{0}, g_{0}\right)$. Let $\left(E_{0}, \nabla_{0}\right)$ and $\left(E_{z}, \nabla_{z}\right)$ be rank $k$ Hermitian vector bundles over $\left(M_{0}, g_{0}\right)$ and $\left(Z, g_{z}\right)$, respectively, so that each of these bundles in a neighborhood of the boundary is the pullback from a bundle over the boundary $(Y, h)$. Let $\Delta_{0}, \Delta_{z}$ be the corresponding Friedrichs extensions of geometric Laplacians, and let $\Delta_{\epsilon}$ be the induced geometric Laplacian on $\left(M, g_{\epsilon}\right)$. Assume $\Delta_{z}$ has no $\mathcal{L}^{2}$ nullspace. Then the accumulation points of the spectrum of $\Delta_{\epsilon}$ as $\epsilon \rightarrow 0$ are precisely the points of the spectrum of $\Delta_{0}$, counting multiplicity.

The setting for our next result is the acc heat space, a manifold with corners constructed in Section 7. Note that the hypotheses for this result are weaker: $\Delta_{z}$ may have nontrivial $\mathcal{L}^{2}$ cohomology and the dimension may be 2 or greater.

Theorem 7.2. Let $\left(M_{0}, g_{0}\right)$ be a compact Riemannian $n$-manifold with isolated conic singularity, and let $\left(Z, g_{z}\right)$ be an ac space, with $n \geq 2$. Assume $\left(M, g_{\epsilon}\right)$ converges asymptotically conically to $\left(M_{0}, g_{0}\right)$. Let $\left(E_{0}, \nabla_{0}\right)$ and $\left(E_{z}, \nabla_{z}\right)$ be rank $k$ Hermitian vector bundles over $\left(M_{0}, g_{0}\right)$ and $\left(Z, g_{z}\right)$, respectively, so that each of these bundles in a neighborhood of the boundary is the pullback from a bundle over the boundary $(Y, h)$. Let $\Delta_{0}$ and $\Delta_{z}$ be the corresponding Friedrichs extensions of geometric Laplacians, and let $\Delta_{\epsilon}$ be the induced geometric Laplacian on $\left(M, g_{\epsilon}\right)$. Then the associated heat kernels $H_{\epsilon}$ have a full polyhomogeneous expansion as $\epsilon \rightarrow 0$ on the acc heat space with the following leading terms:

- At the conic front face, $F_{0101}, H_{\epsilon}\left(z, z^{\prime}, t\right) \rightarrow H_{0}\left(z, z^{\prime}, t\right)$, the heat kernel for $\left(M_{0}, g_{0}\right)$.

- At the rescaled $b$ front face, $F_{1010,2}, H_{\epsilon}\left(z, z^{\prime}, t\right) \rightarrow\left(\rho_{1010,2}\right)\left(\rho_{1010}\right)$ $\left(\rho_{0101}\right)^{-1} H_{b}(\tau)$, the $b$ heat kernel with rescaled time variable $\tau$.

- At the exact conic front face, $F_{1111,2}, H_{\epsilon}\left(z, z^{\prime}, t\right) \rightarrow H_{0}(\widetilde{\tau})$, the heat kernel for the exact cone with rescaled time variable $\widetilde{\tau}$.

- At the side faces $F_{1001}, F_{0110}$ and the residual $b$ face $F_{1010}$, the heat kernel has polyhomogeneous expansion with leading term vanishing to at least second order.

This convergence is uniform in $\epsilon$ for bounded time and moreover, the error term is bounded by $C \epsilon t^{N}$ as $t \rightarrow 0$, for any $N \in \mathbb{N}_{0}$, where $C$ may depend on $N$. 


\section{Remarks.}

- This theorem immediately implies the uniform convergence

$$
H_{\epsilon}\left(z, z^{\prime}, t\right) \rightarrow H_{0}\left(z, z^{\prime}, t\right), \quad T>t>0
$$

as well as the convergence

$$
H_{\epsilon}\left(z, z^{\prime}, t\right) \rightarrow H_{0}\left(z, z^{\prime}, t\right)+O(\epsilon), \quad t, \epsilon \rightarrow 0
$$

with explicit error given by the leading terms above in the polyhomogeneous expansion of $H_{\epsilon}\left(z, z^{\prime}, t\right)$ on the acc heat space as $\epsilon \rightarrow 0$.

- We have dropped the half-density factor,

$$
\left(\rho_{1111,2}\right)^{n+1}\left(\rho_{1010,2}\right)^{n}\left(\rho_{1010}\right)^{n-1}\left(\rho_{d 2}\right)^{(n+1) / 2}\left(\rho_{1001} \rho_{0110}\right)^{(n-1) / 2} \sqrt{\nu},
$$

where above $\nu$ is a smooth non-vanishing density on the acc heat space.

These theorems are proven in Sections 5 and 7, respectively. In Section 2, we define the resolution blowup and ac convergence. Sections 3 and 4 contain a brief review of geometric and analytic results and terminology on manifolds with corners. In Section 6, we construct the heat spaces and heat operator calculi that will be used to prove the main theorem in Section 7.

This work is based on the author's doctoral dissertation completed at Stanford University in June 2006 under the supervision of Rafe Mazzeo. The author wishes to thank Rafe Mazzeo for excellent advising, Andras Vasy for many helpful conversations and suggestions, Richard Melrose for enlightening conversations and correspondence and the anonymous referee for useful comments.

\section{Asymptotically conic convergence}

The conic collapse in ac convergence is locally modeled by an ac scattering metric or ac space. The following definitions, which may be used differently by other authors, were motivated to impose as few restrictions on the geometry of the collapse as possible while simultaneously maintaining sufficient control to uniformly analyze the Laplacian and its heat kernel.

Definition 2.1. An $a c$ scattering metric $\left(\bar{Z}, g_{z}\right)$ is a smooth complete metric on a compact manifold with boundary so that a neighborhood of the 
boundary is diffeomorphic to a product $\left(0, r_{1}\right)_{r} \times Y$, where $r$ is a boundarydefining function for the boundary $Y$. On this neighborhood,

$$
g_{z} \sim \frac{d r^{2}}{r^{4}}+\frac{h(r)}{r^{2}}, \quad h(r) \sim h_{0}+r h_{1}+r^{2} h_{2}+\cdots, \text { as } r \rightarrow 0,
$$

where $\left\{h_{k}\right\}_{k \geq 0}$ are a smooth family of Riemannian metrics on $Y .{ }^{3}$ Uniquely associated to the ac scattering metric $\left(\bar{Z}, g_{z}\right)$ is the complete non-compact manifold $Z$ with ac end; $Z$ is known as an ac space. ${ }^{4}$ Letting $\rho=\frac{1}{r}$, there is a compact subset $K_{z} \subset Z$ so that

$$
Z-K_{z} \cong\left(\frac{1}{r_{1}}, \infty\right)_{\rho} \times Y,\left.\quad g_{z}\right|_{\left(Z-K_{z}\right)}=d \rho^{2}+\rho^{2} h(1 / \rho) .
$$

A familiar example of an ac scattering metric is the radial compactification of $\mathbb{R}^{n}$ with boundary $\mathbb{S}^{n-1}$. The intuitive geometric picture of an ac space is the "big end of a complete cone".

Definition 2.2. Let $M$ be a compact metric space with Riemannian metric $g$. Then, $(M, g)$ has an isolated conical singularity at the point $p$ and $g$ is called a conic metric if

1. $(M-\{p\}, g)$ is a smooth, open manifold.

2. There is $x_{1}>0$ and a neighborhood $N$ of $p$ with a function $x:(N-$ $\{p\}) \rightarrow\left(0, x_{1}\right]$ such that $N-\{p\}$ is diffeomorphic to $\left(0, x_{1}\right]_{x} \times Y$ and

$$
g \sim d x^{2}+x^{2} h(x), \quad h(x) \sim h_{0}+x h_{1}+x^{2} h_{2}+\ldots, \text { as } x \rightarrow 0,
$$

where $\left\{h_{k}\right\}_{k \geq 0}$ are a smooth family of metrics on $Y$.

By "blowing up" the cone point $p$ adding a copy of $\left(Y, h_{0}\right)$ at $x=0$, a conic metric is an incomplete metric on a compact manifold with boundary whose metric has the above degenerate form in a neighborhood of the boundary defined by $\{x=0\}$.

\footnotetext{
${ }^{3}$ For technical reasons, we require the stronger assumption that $h$ has a formal power series at the boundary rather than the standard regularity assumption that $h(r)$ extends smoothly to a metric on the boundary.

${ }^{4}$ Note that ac spaces are sometimes called "asymptotically locally Euclidean", or $A L E$. However, that term is often used for the more restrictive class of spaces that are asymptotic at infinity to a cone over a quotient of the sphere by a finite group, so to avoid confusion, we use the term asymptotically conic.
} 
The intuitive geometric picture of a conic metric is an open manifold, with a boundary that pinches to the point of a cone. It is an incomplete metric because the cone point is not included in the smooth Riemannian structure.

In the definition of resolution blowup, we use the notation $M \cup_{\phi} N$ for a smooth manifold constructed from the smooth manifolds $M$ and $N$ with a diffeomorphism $\phi$ from $V \subset N$ to $U \subset M$ that gives the equivalence relation, $V \ni p \sim \phi(p) \in U . M \cup_{\phi} N$ is the disjoint union of $M$ and $N$ modulo the equivalence relation of $\phi$. The smooth structure on $M \cup_{\phi} N$ and the topology are induced by those of $M$ and $N$.

Definition 2.3. Let $\left(M_{0}, g_{0}\right)$ be a compact $n$ dimensional manifold with isolated conic singularity and let $\left(Z, g_{z}\right)$ be an ac space of dimension $n$, so that $\partial M_{0}=\partial \bar{Z}=\left(Y, h_{0}\right)$. Then, the interior of $M_{0}$,

$$
M_{0}^{0}=K_{0} \cup V_{0},
$$

where $V_{0} \cong\left(0, x_{1}\right)_{x} \times Y$, and $K_{0}$ is compact. With this diffeomorphism

$$
g_{0}=d x^{2}+x^{2} \tilde{h}(x) \quad \text { on }\left(0, x_{1}\right)_{x} \times Y,
$$

and the boundary of $K_{0}$ in $M_{0}$ is of the form $\partial K_{0}=\left\{x=x_{1}\right\} \cong Y$. Similarly,

$$
Z=K_{z} \cup V_{z}
$$

where $V_{z} \cong\left(\rho_{1}, \infty\right)_{\rho} \times Y$, and $K_{z}$ is compact. With this diffeomorphism,

$$
g_{z}=d \rho^{2}+\rho^{2} h(\rho, y) \quad \text { on }\left(\rho_{1}, \infty\right)_{\rho} \times Y
$$

and the boundary of $K_{z}$ in $Z$ is of the form $\partial K_{z}=\left\{\rho=\rho_{1}\right\} \cong Y$.

Let $\delta=\min \left\{x_{1}, 1 / \rho_{1}\right\}$. Then for $0<\epsilon<\delta$, and $R>\frac{1}{\delta}$, let

$$
M_{0, \epsilon}=\left\{(x, y) \in M_{0}: x>\epsilon\right\} \cup K_{0}, \quad Z_{R}=\{(\rho, y) \in Z: \rho<R\} \cup K_{z} .
$$

The resolution blowup of $\left(M_{0}, g_{0}\right)$ by $\left(Z, g_{z}\right)$ is

$$
M_{\epsilon}:=M_{0, \epsilon} \cup_{\phi_{\epsilon}} Z_{1 / \epsilon},
$$

where the joining map $\phi$ is defined for each $\epsilon$ by

$$
\phi_{\epsilon}: M_{0, \epsilon}-M_{0, \delta} \rightarrow Z_{1 / \epsilon}-Z_{1 / \delta}, \quad \phi_{\epsilon}(x, y)=\left(\frac{x}{\delta \epsilon}, y\right) .
$$


For $\delta>\epsilon>\epsilon^{\prime}>0$, the manifolds $M_{\epsilon}$ and $M_{\epsilon^{\prime}}$ are diffeomorphic, and so the resolution blowup of $M_{0}$ by $Z$, which we call $M$, is unique up to diffeomorphism.

Remark. The resolution blowup is a smooth compact manifold which resolves a conical singularity using an ac space. Instead of resolving the singularity in $M_{0}$ using $Z$, we may equivalently define the resolution blowup to resolve the boundary of $\bar{Z}$ using $M_{0}$ as follows. Let $r=1 / \rho$ be the defining function for $\partial \bar{Z}$. The resolution blowup of $\bar{Z}$ by $M_{0}$ is

$$
M_{\epsilon}:=M_{0, \epsilon} \cup_{\psi_{\epsilon}} Z_{1 / \epsilon}, \quad \psi_{\epsilon}(x, y)=\left(\frac{\epsilon \delta}{x}, y\right),
$$

where the joining map $\psi_{\epsilon}: M_{0, \epsilon}-M_{0, \delta} \rightarrow Z_{1 / \epsilon}-Z_{1 / \delta}$ is defined by $\psi_{\epsilon}(x, y)$ $=(\epsilon \delta / x, y)=(r, y)$. The resulting smooth compact resolution space is diffeomorphic to $M$.

The ac single space, analogous to the analytic surgery single space in [30], is the setting for the definition of ac convergence.

Definition 2.4. Let $\left(M_{0}, g_{0}\right)$ be a conic metric and let $\left(\bar{Z}, g_{z}\right)$ be a scattering metric; assume both are dimension $n$ with $\partial M_{0}=\partial \bar{Z}=(Y, h)$, and assume $\delta=1$ (Definition 2.3). Then, $M_{0} \cong\left((0,1)_{x} \times Y\right) \cup K_{0}$ and $\bar{Z} \cong$ $\left((0,1)_{r} \times Y\right) \cup K_{z}$ with $\partial K_{0} \cong Y \cong \partial K_{z}$. The acc single space $\mathcal{S}$ is

$$
\mathcal{S}:=[0,1)_{x} \times[0,1)_{r} \times Y \cup\left(K_{0} \times\{x=1, r \neq 1\}\right) \cup\left(K_{z} \times\{r=1, x \neq 1\}\right) .
$$

The smooth structure of $\mathcal{S}$ is induced by that of $M_{0}$ and $\bar{Z}$. Namely, smooth functions on $\mathcal{S}$ are functions which are smooth jointly in $x$ and $r$ on $(0,1)_{x} \times(0,1)_{r} \times Y$ and smoothly extend to a smooth function on $K_{0}$ at $x=1$, on $K_{z}$ at $r=1$, on $M_{0}$ at $r=0$ and on $\bar{Z}$ at $x=0$.

Definition 2.5. Let $\mathcal{S}$ be the acc single space associated to $M_{0}$ and $\bar{Z}$ as in Definition 2.4. Let $\epsilon(p)=x(p) r(p): \mathcal{S} \rightarrow[0,1)$, where $x$ and $r$ are extended to $K_{0}$ and $K_{z}$, respectively, to be identically 1 . We define the acc tensor $\mathcal{G}$ as follows:

$$
\mathcal{G}= \begin{cases}\frac{1}{2}\left(d x^{2}+x^{2}\left(h(x)+r^{2}\left(\frac{d r^{2}}{r^{4}}+\frac{h(r)}{r^{2}}\right)\right)\right), & x, r \in(0,1), \\ (x r)^{2}\left(\left.g_{z}\right|_{K_{z}}\right), & r=1, \\ \left.g_{0}\right|_{K_{0}}, & x=1 .\end{cases}
$$


For $0<\epsilon<1$, let $M_{\epsilon}=\{x r=\epsilon\} \subset \mathcal{S}$; note that $M_{\epsilon}$ is diffeomorphic to the resolution blowup $M$ of $M_{0}$ by $Z$. The family of metrics $\left\{g_{\epsilon}=\left.\mathcal{G}\right|_{M_{\epsilon}}\right\}$ on $M$ is said to converge asymptotically conically to $\left(M_{0}, g_{0}\right)$.

\section{Remarks.}

1. In the definition of the acc single space to simplify calculations, we have assumed $\delta=1$; no generality is lost by this assumption. On $\{x r=\epsilon\}$, we then have $r=\frac{\epsilon \delta}{x}$, equivalently $\rho=\frac{1}{r}=\frac{x}{\epsilon \delta}$. Letting $\delta=1$ in the definition of resolution blowup, the identification of $\{x r=\epsilon\} \subset \mathcal{S}$ with the resolution blowup $M_{\epsilon}$ follows immediately.

2. The acc single space has two boundary hypersurfaces at $\epsilon=0$. These are diffeomorphic to $M_{0}$ at $r=0$ and $\bar{Z}$ at $x=0$, and they meet in a codimension 2 corner diffeomorphic to $Y$.

3. The acc single space contains a submanifold diffeomorphic to a cone over $[0,1)_{r} \times Y \subset \bar{Z}$ with radial variable $x$.

4. At $r=0, \mathcal{G}$ restricts to $\left.\mathcal{G}\right|_{\{r=0\}}=g_{0}$. On $M_{\epsilon} \subset \mathcal{S}$ where $0<r(p)$, $x(p)<1$,

$$
r=\frac{\epsilon}{x} \quad \Longrightarrow d r^{2}=\frac{\epsilon^{2}}{x^{4}} d x^{2} \quad \Longrightarrow g_{\epsilon}=d x^{2}+x^{2} h(x),
$$

which shows that $g_{\epsilon}$ extends smoothly to $\left.g_{0}\right|_{K_{0}}$ as $x \rightarrow 1$. Similarly, we compute

$$
g_{\epsilon}=\epsilon^{2}\left(\frac{d r^{2}}{r^{4}}+\frac{h(r)}{r^{2}}\right)
$$

so $g_{\epsilon}$ extends smoothly to $\left(\epsilon^{2}\right)\left(\left.g_{z}\right|_{K_{z}}\right)$ as $r \rightarrow 1$. $\mathcal{G}$ is a smoothly polyhomogeneous symmetric 2-cotensor on $\mathcal{S}$, and its restriction $g_{\epsilon}$ is also smooth and polyhomogeneous as $\epsilon \rightarrow 0$.

The following lemma is useful for visualizing ac convergence and for proving spectral convergence.

Lemma 2.6. Let $\left(M_{0}, g_{0}\right)$ and $\left(Z, g_{z}\right)$ be as in Definitions 2.3 to 2.5 and let $\left(M, g_{\epsilon}\right)$ converge asymptotically conically to $\left(M_{0}, g_{0}\right)$. Then, there exists a family of diffeomorphisms $\left\{\phi_{\epsilon}\right\}$ from a fixed open proper subset $U \subset M$ to increasing neighborhoods $Z_{1 / \epsilon} \subset Z$ such that $\left.\left.g_{\epsilon}\right|_{U} \cong\left(\epsilon^{2}\left(\phi_{\epsilon}\right)^{*}\left(\left.g\right|_{Z_{1 / \epsilon}}\right)\right)\right|_{U}$. Moreover, on $M-U, g_{\epsilon} \rightarrow g_{0}$ smoothly as $\epsilon \rightarrow 0$, and any $K \subset \subset M_{0}^{0}$ is diffeomorphic to some fixed $K^{\prime} \subset M$ so that $g_{\epsilon} \rightarrow g_{0}$ smoothly and uniformly on $K^{\prime}$. 
Proof. The existence of $\phi_{\epsilon}$ and $U \subset M$ follows immediately from the definition of resolution blowup and the diffeomorphism between the resolution blowup $M$ and $\{x r=\epsilon\} \subset \mathcal{S}$. By the above remarks, on the neighborhood $U \subset M$ where this diffeomorphism is defined, $\left.g_{\epsilon}\right|_{U}=\left(\epsilon^{2}\right)\left(\phi_{\epsilon}\right)^{*}\left(\left.g_{z}\right|_{Z_{1 / \epsilon}}\right)$.

Since $g_{\epsilon}=g_{0}+\mathcal{O}\left(\epsilon^{2}\right)$, the smooth convergence of $g_{\epsilon}$ to $g_{0}$ on $M-U$ follows immediately. Any compact subset $K \subset \subset M_{0}$ is contained in $M_{0, \epsilon}$ for some $\epsilon>0$ and so is diffeomorphic to $K_{\epsilon} \subset \subset M_{\epsilon}$ and also to $K^{\prime} \subset \subset$ $\left(M-U^{\prime}\right)$. Conversely, any $K \subset \subset(M-U)$ is diffeomorphic to $K_{\epsilon} \subset M_{\epsilon}$ for some $\epsilon>0$ and also to $K^{\prime} \subset \subset M_{0, \epsilon} \subset M_{0}$.

\section{Geometric preliminaries}

This section is a brief review of the theory and terminology of manifolds with corners, $b$ maps, and blowups; see also $[28,33]$.

\subsection{Manifolds with corners}

Let $X$ be a manifold with corners. This means that near any of its points, $X$ is modeled on a product $[0, \infty)^{k} \times \mathbb{R}^{n-k}$, where $k$ depends on the point and is the maximal codimension of the boundary face containing that point. We also assume that all boundary faces of $X$ are embedded so they too are manifolds with corners. The space $\mathcal{V}(X)$ of all smooth vector fields on $X$ is a Lie algebra under the standard bracket operation. It contains the Lie subalgebra

(3.1) $\mathcal{V}_{b}(X):=\{V \in \mathcal{V}(X): V$ is tangent to each boundary face of $X\}$.

Then $\mathcal{V}_{b}(X)$ is itself the space of all smooth sections of a vector bundle,

$$
\mathcal{V}_{b}(X)=\mathcal{C}^{\infty}\left(X ;{ }^{b} T X\right),
$$

where ${ }^{b} T X$ is the bundle defined so that the above holds and is called the $b$-tangent bundle.

3.1.1. Blowing up. An embedded codimension $k$ submanifold $Y$ of a manifold with corners $X$ is called a $p$-submanifold ( $p$ for product) if near each point of $Y$ there are local product coordinates so that $Y$ is defined by the vanishing of some subset of them. In other words, $X$ and $Y$ must have consistent local product decompositions. Then one can define a new manifold with corners $[X ; Y]$ to be the normal blowup of $X$ around $Y$. This is 
obtained by replacing $Y$ by its inward-pointing spherical normal bundle. The union of this normal bundle and $X-Y$ has a unique minimal differential structure as a manifold with corners so that the lifts of smooth functions on $X$ and polar coordinates around $Y$ are smooth. One can also consider iterated blowups, written $\left.\left[\left[X ; Y_{1}\right] ; Y_{2}\right]\right]$, where $Y_{1}$ and $Y_{2}$ are $p$-submanifolds of $X$. However, depending on the geometry, this may not yield the same space as $\left[\left[X ; Y_{2}\right] ; Y_{1}\right]$. In the special case of embedded $p$-submanifolds,

$$
X \supset Y_{1} \supset Y_{2} \supset Y_{3} \supset \cdots \supset Y_{n}
$$

the iterated blowup

$$
\left.\left[\left[X ; Y_{1}\right] ; Y_{2}\right] ; \cdots ; Y_{n}\right]
$$

can be performed in any order with the same result [30]. Blowups may also be defined using equivalence classes of curves [33]. Let $r$ be a defining function for the $p$ submanifold $Y$ and consider the family of curves $\gamma(t)=$ $(r(t), y(t))$ such that

$$
\begin{gathered}
\gamma(t) \in Y \Longleftrightarrow t=0 \\
r(t)=O(t)
\end{gathered}
$$

Let $E$ be the set of equivalence classes of all such curves with

$$
\gamma \sim \gamma^{\prime} \Longleftrightarrow\left(y-y^{\prime}\right)(t)=O(t) \quad \text { and } \quad\left(r-r^{\prime}\right)(t)=O\left(t^{2}\right)
$$

There is a natural $\mathbb{R}^{+}$action on $E$ given by

$$
\mathbb{R}^{+} \ni a: \gamma(t) \rightarrow \gamma(a t)
$$

$E$ modulo this equivalence relation is naturally diffeomorphic to $N^{+}(Y)$, the inward-pointing spherical normal bundle of $Y$, so we can define $[X ; Y]$ by

$$
[X ; Y]=(X-Y) \cup E /\left(\mathbb{R}^{+}-\{0\}\right)
$$

We can also define parabolic blowups in certain contexts [12]. Let $Y$ be a $p$-submanifold of codimension $k$ so that there exist local coordinates $(r, y)=$ $\left(r_{1}, \ldots, r_{k}, y_{1}, \ldots, y_{n-k}\right)$ in a neighborhood of $Y$ with $r_{i}$ vanishing precisely at $Y$ and so that $d r_{1}$ induces a sub-bundle of the tangent bundle $T X$. Instead 
of the above equivalence classes of curves, we consider $\gamma(t)$ such that

$$
\begin{gathered}
\gamma(t)=\left(r_{1}(t), \ldots, r_{k}(t), y_{1}(t), \ldots y_{n-k}(t)\right) \in Y \Longleftrightarrow t=0, \\
r_{i}(t)=O(t), \quad i \neq 1, \quad r_{1}(t)=O\left(t^{2}\right) .
\end{gathered}
$$

Two such curves are equivalent if

$$
\begin{aligned}
& \gamma \sim \gamma^{\prime} \Longleftrightarrow\left(y_{j}-y_{j}^{\prime}\right)(t)=O(t), \quad\left(r_{i}-r_{i}^{\prime}\right)(t)=O\left(t^{2}\right) i \neq 1, \\
&\left(r_{1}(t)-r_{1}^{\prime}(t)\right)=O\left(t^{3}\right) .
\end{aligned}
$$

Since $d r_{1}$ is a sub-bundle of $T X$, there is a natural $\mathbb{R}^{+}$action on the set of equivalence classes $E_{2}$ of all such curves,

$$
\mathbb{R}^{+} \ni a: \gamma(t) \rightarrow\left(r_{1}\left(a^{2} t\right), r_{i}(a t), \ldots, y_{j}(a t)\right) .
$$

The set of equivalence classes of all such curves modulo this $\mathbb{R}^{+}$action is naturally diffeomorphic to the inward-pointing $r_{1}$-parabolic normal bundle of $Y$,

$$
E_{2} /\left(\mathbb{R}^{+}-\{0\}\right) \cong P N_{r_{1}}^{+}(Y) .
$$

We define the $r_{1}$-parabolic blowup of $X$ around $Y$ as the union of $X-Y$ and this inward-pointing $r_{1}$-parabolic bundle,

$$
\left[X ; Y, d r_{1}\right]:=(X-Y) \cup P N_{r_{1}}^{+}(Y) .
$$

The union of this $r_{1}$-parabolic bundle and $X-Y$ again has a unique minimal differential structure as a manifold with corners so that the lifts of smooth functions on $X$ and $r_{1}$-parabolic coordinates around $Y$ are smooth. By $r_{1}$-parabolic coordinates around $Y$, we mean the coordinates,

$$
\rho=\left(r_{1}^{2}+r_{2}^{4}+\cdots+r_{k}^{4}\right)^{1 / 4}, \quad \theta=\left(\theta_{1}, \ldots, \theta_{n}\right) \in \mathbb{S}^{n-1},
$$

with local coordinates $\left(r_{1}, \ldots, r_{k}, y_{1}, \ldots, y_{n-k}\right)$ in a neighborhood of $Y$ satisfying

$$
r_{i}=\rho \theta_{i}, \quad i \neq 1, \quad r_{1}=\rho^{2} \theta_{1}, \quad y_{j}=\rho \theta_{j} .
$$

For any parabolic or spherical blowup, there is a natural blow-down map $\beta_{*}:[X ; Y] \rightarrow X$ and corresponding blowup map $\beta^{*}: X \rightarrow[X ; Y]$, so that the image of $Y$ under $\beta^{*}$ is a boundary hypersurface of $[X ; Y]$ diffeomorphic to the inward-pointing spherical (or parabolic) normal bundle of $Y$. As such,

$$
[X ; Y]=(X-Y) \cup \beta^{*}(Y) .
$$




\subsection{2. $b$-Maps and $b$-fibrations}

Definition 3.1. Let $M_{1}$ be a manifold with boundary hypersurfaces, $\left\{N_{j}\right\}_{j=1}^{k}$, and defining functions $r_{j}$. Let $M_{2}$ be a manifold with boundary hypersurfaces, $\left\{L_{i}\right\}_{i=1}^{l}$, and defining functions $\rho_{i}$. Then $f: M_{1} \rightarrow M_{2}$ is called a $b$-map if for every $i$ there exist non-negative integers $e(i, j)$ and a smooth non-vanishing function $h$ such that $f^{*}\left(\rho_{i}\right)=h \prod_{j=1}^{k} r_{j}^{e(i, j)}$.

The image under a $b$-map of the interior of each boundary hypersurface of $M_{1}$ is either contained in or disjoint from each boundary hypersurface of $M_{2}$, and the order of vanishing of the differential of $f$ is constant along each boundary hypersurface of $M_{1}$. The matrix $(e(i, j))$ is called the lifting matrix for $f$.

In order for the map $f$ to preserve polyhomogeneity, stronger conditions are required. Associated to a manifold with corners are the $b$-tangent and cotangent bundles, ${ }^{b} T M(3.1)$ and ${ }^{b} T^{*} M{ }^{5}$ The map $f$ may be extended to induce the map ${ }^{b} f_{*}: b T M_{1} \rightarrow^{b} T M_{2}$.

Definition 3.2. The $b$-map, $f: M_{1} \rightarrow M_{2}$, is called a $b$-fibration if the associated maps ${ }^{b} f_{*}$ at each $p \in \partial M_{1}$ are surjective at each $p \in \partial M_{1}$, and the lifting matrix $(e(i, j))$ has the property that for each $j$ there is at most one $i$ such that $(e(i, j)) \neq 0$. In other words, $f$ does not map any boundary hypersurface of $M_{1}$ to a corner of $M_{2}$.

3.1.3. $b$-Manifolds and the $b$-blowup A $b$-manifold is a complete manifold with boundary whose metric is closely related to conic and ac scattering metrics.

Definition 3.3. Let $(X, g)$ be a smooth Riemannian manifold with boundary $(Y, h)$ and boundary-defining function $x$ so that a neighborhood of the boundary is diffeomorphic to a product $\left[0, x_{1}\right)_{x} \times Y$ and in this neighborhood

$$
g=\frac{d x^{2}}{x^{2}}+h(x)
$$

where $h(x)$ is a smoothly varying family of metrics on $Y$ that converges smoothly to $h$ as $x \rightarrow 0$. Then $(X, g)$ is said to be a $b$-manifold.

Equivalently, under the change of variables $r \mapsto e^{-t}$, a $b$-manifold is seen to be the compactification as a manifold with boundary of a complete manifold with asymptotically cylindrical end(s). To study the regularity and

\footnotetext{
${ }^{5}$ These are also called the totally characteristic tangent and cotangent bundles.
} 


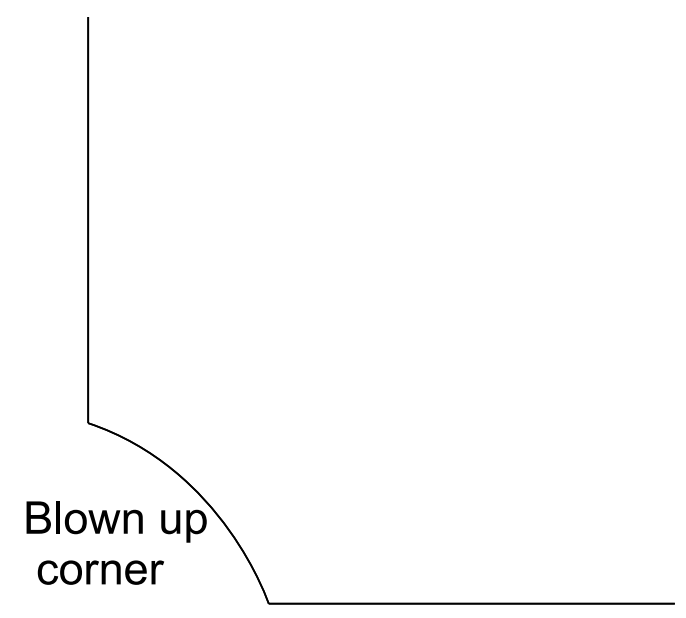

Figure 1: The $b$-blowup $X_{b}^{2}$.

mapping properties of geometric operators on $b$-manifolds, melrose [33] introduced the $b$-double space. This space is obtained from $X^{2}$ by performing a radial blowup called the $b$-blowup along the codimension 2 corner at the boundary in each copy of $X$, and it is written $X_{b}^{2}$ (Figure 1),

$$
X_{b}^{2}=[X \times X ; \partial X \times \partial X]=[X \times X ; Y \times Y]
$$

For any manifold $M$ with boundary having a product structure in a neighborhood of the boundary, we may define the $b$-blowup in the analogous way, $M_{b}^{2}:=[M \times M ; \partial M \times \partial M]$.

\subsection{Asymptotically conic convergence double space}

The acc double space is an instructive model for the more complicated acc heat space in Section 7. Let

$$
\mathcal{S}_{b}^{2}:=\left[\mathcal{S}^{2} ; Y \times Y\right]
$$

The acc double space $\mathcal{D}$ is the submanifold of $\mathcal{S}_{b}^{2}$ defined by the vanishing set of

$$
f(p)=x(p) r(p)-x^{\prime}(p) r^{\prime}(p)=\epsilon(p)-\epsilon^{\prime}(p),
$$

so that

$$
\mathcal{D}=\left\{p \in \mathcal{S}_{b}^{2}: f(p)=x(p) r(p)-x^{\prime}(p) r^{\prime}(p)=0\right\}=\left\{p \in \mathcal{S}_{b}^{2}: \epsilon(p)=\epsilon^{\prime}(p)\right\}
$$


The acc double space has four boundary faces at $\epsilon=0$, described in the following table. Here and throughout, we label each face $F_{w x y z}$ where the subscript indicates the order to which each of the scalar variables $x, r, x^{\prime}, r^{\prime}$ vanishes at that face.

\begin{tabular}{lll}
\hline Scalar variables & Face & Geometry \\
\hline$x=0, x^{\prime}=0$, & $F_{1010}$ & {$[\bar{Z} \times \bar{Z} ; Y \times Y]$} \\
$x=0, r^{\prime}=0$ & $F_{1001}$ & {$\left[\bar{Z} \times M_{0} ; Y \times Y\right]$} \\
$r=0, x^{\prime}=0$ & $F_{0110}$ & {$\left[M_{0} \times \bar{Z} ; Y \times Y\right]$} \\
$r=0, r^{\prime}=0$ & $F_{0101}$ & {$\left[M_{0} \times M_{0} ; Y \times Y\right]$} \\
\hline
\end{tabular}

To see that $\mathcal{D}$ is a smooth submanifold of $\mathcal{S}_{b}^{2}$, we consider the function

$$
f(p)=x(p) r(p)-x^{\prime}(p) r^{\prime}(p) .
$$

Away from the boundary faces, $f$ is smooth with non-vanishing differential. In a neighborhood of $S_{11}-F_{1001}$, let

$$
f_{0110}(p)=\frac{x(p)}{x^{\prime}(p)}-\frac{r^{\prime}(p)}{r(p)} .
$$

Since $x^{\prime} r f_{0110}=f$, we see that $f_{0110}$ is smooth near the $\epsilon=0$ boundary faces away from where those faces meet $F_{0110}$. Moreover, wherever defined, $f_{0110}$ has non-vanishing differential and its zero set coincides with that of $f$ away from $F_{0110}$. Similarly, let

$$
f_{1001}(p)=\frac{x(p)}{r^{\prime}(p)}-\frac{x^{\prime}(p)}{r(p)} .
$$

$f_{1001}$ is smooth with non-vanishing differential and has the same vanishing set as $f$ in a neighborhood of $\{\epsilon=0\}-F_{1001}$. This shows that $\mathcal{D}$ is a smooth submanifold of $\mathcal{S}_{b}^{2}$. While the acc double space will not be used here, we note that the acc double space, with an additional blowup along the diagonal for $\epsilon \geq 0$, would be the natural space on which to study the resolvent behavior under ac convergence.

\section{Analytic preliminaries}

Since we are working on manifolds with singularities corners and boundaries, we briefly review some key features of the analysis in these settings. 


\subsection{Polyhomogeneous conormal functions}

On a manifold $M$ with corners having a consistent local product structure near each boundary and corner, a natural class of functions (or sections) with good regularity near the boundary and corners are the polyhomogeneous conormal functions (or sections); see [28]. In a neighborhood of a corner, we have coordinates $\left(x_{1}, \ldots, x_{k}, y_{1}, \ldots, y_{n-k}\right)$, where $x_{1}, \ldots, x_{k}$ vanish at this corner and $\left(y_{1}, \ldots, y_{n-k}\right)$ are smooth local coordinates on a smooth compact $n-k$ manifold $Y$. The edge tangent bundle $\mathcal{V}_{e}$ in a neighborhood of this corner is spanned over $\mathcal{C}^{\infty}(M)$ by the vector fields,

$$
\left\{x_{i} \partial_{x_{i}}, \partial_{y^{\alpha}}\right\}
$$

The basic conormal space of sections is

$$
\mathcal{A}^{0}\left(M_{0}\right)=\left\{\phi: V_{1} \cdots V_{l} \phi \in L^{\infty}\left(M_{0}\right), \forall V_{i} \in \mathcal{V}_{e}, \text { and } \forall l\right\} .
$$

Let $\alpha$ and $p$ be multi-indices with $\alpha_{j} \in \mathbb{C}$ and $p_{j} \in \mathbb{N}_{0}$. Then we define

$$
\mathcal{A}^{\alpha, p}\left(M_{0}\right)=x^{\alpha}(\log x)^{p} \mathcal{A}^{0} .
$$

The space $\mathcal{A}^{*}$ is the union of all these spaces for all $\alpha$ and $p$. The space $\mathcal{A}_{\text {phg }}^{*}\left(M_{0}\right)$ consists of all conormal distributional sections which have an expansion of the form

$$
\phi \sim \sum_{\operatorname{Re}\left(\alpha_{j}\right) \rightarrow \infty} \sum_{p=0}^{p_{j}} x^{\alpha_{j}}(\log x)^{p} a_{j, p}(x, y), \quad a_{j, p} \in \mathcal{C}^{\infty} .
$$

We define an index set to be a discrete subset $E \subset \mathbb{C} \times \mathbb{N}_{0}$ such that

$$
\left(\alpha_{j}, p_{j}\right) \in E, \quad\left|\left(\alpha_{j}, p_{j}\right)\right| \rightarrow \infty \Longrightarrow \operatorname{Re}\left(\alpha_{j}\right) \rightarrow \infty .
$$

Then, the space $\mathcal{A}_{p h g}^{E}\left(M_{0}\right)$ consists of those distributional sections $\phi \in \mathcal{A}_{p h g}^{*}$ having polyhomogeneous expansions with $\left(\alpha_{j}, p_{j}\right) \in E$.

\subsection{Conic differential operators and $b$-operators}

Let $\left(M_{0}, g_{0}\right)$ be a Riemannian manifold with isolated conic singularity so that in a neighborhood of the singularity,

$$
M_{0} \cong\left(0, x_{1}\right)_{x} \times Y, \quad g_{0}=d x^{2}+x^{2} h(x) .
$$


A conic differential operator of order $m$ is a smooth differential operator on $M_{0}$ which can be expressed in neighborhood of the singularity by

$$
A=x^{-m} \sum_{k=0}^{m} B_{k}(x)\left(-x \partial_{x}\right)^{k}
$$

with $B_{k} \in \mathcal{C}^{\infty}\left(\left(0, x_{1}\right)\right.$, Diff $\left.{ }^{m-k}(Y)\right)$, where $\operatorname{Diff}^{j}(Y)$ denotes the space of differential operators of order $j \in \mathbb{N}_{0}$ on $Y$ with smooth coefficients. The cone differential operators are elements of the cone operator calculus; for a detailed description, see [24]. These cone operators are closely related to $b$-operators. A $b$-operator of order $m$ is a smooth differential operator such that near the boundary it can be expressed by

$$
A=\sum_{k=0}^{m} B_{k}(x)\left(-x \partial_{x}\right)^{k}
$$

with $B_{k} \in \mathcal{C}^{\infty}\left(\left(0, x_{1}\right)\right.$, $\left.\operatorname{Diff}^{m-k}(Y)\right)$. Note that a cone differential operator of order $m$ is equal to a rescaled $b$-differential operator of order $m$; if $A$ is an order $m$ cone differential operator, then $x^{m} A$ is a $b$-differential operator. In local coordinates $\left(x, y_{1}, \ldots, y_{n-1}\right)$ near the boundary a $b$-operator may be expressed as

$$
A=\sum_{j+|\alpha| \leq m} a_{j, \alpha}(x, y)\left(-x \partial_{x}\right)^{j}\left(\partial_{y^{\alpha}}\right)
$$

The $b$-symbol of $A$ is

$$
{ }^{b} \sigma_{m}(A)=\sum_{j+|\alpha|=m} a_{j, \alpha}(x, y) \lambda^{j} \eta^{\alpha}
$$

Here $\lambda$ and $\eta$ are linear functions on the $b$-cotangent bundle defined by the coordinates so that a generic element of the $b$-cotangent bundle is

$$
\lambda \frac{d x}{x}+\sum_{i=1}^{n} \eta_{i} d y_{i}
$$

The $b$-operator is $b$-elliptic if the $b$-symbol ${ }^{b} \sigma_{m}(A)$ is non-zero off the zero section. 
The scalar Laplacian on $M_{0}$ is

$$
x^{-2}\left\{\left(-x \partial_{x}\right)^{2}+\left(-n+1+x H^{-1}\left(\partial_{x} H\right)\left(-x \partial_{x}\right)\right)+\Delta_{h(x)}\right\}=x^{-2} L_{b},
$$

where $L_{b}$ is an elliptic order $2 b$-operator and $H$ is a smooth function that depends on the metric. Similarly, a geometric Laplacian $\Delta_{0}$ on $M_{0}$ is also of the form

$$
\Delta_{0}=x^{-2} L_{b},
$$

for an elliptic order two $b$-operator acting on sections of the vector bundle. The Schwartz kernel of $L_{b}$ is a distribution on the $b$-double space. By the $b$-calculus theory, [33] $L_{b}$ has a parametrix $G_{b}$ such that $G_{b}$ is a b-operator of order -2 with

$$
G_{b} L_{b}=I-R
$$

where $I$ is the identity operator and $R$ is a $b$-operator with polyhomogeneous Schwartz kernel on the $b$-double space. For any $u \in \mathcal{L}^{2}\left(x^{n-1} d x d y\right)$ with $\Delta_{0} u=f \in \mathcal{L}^{2}\left(x^{n-1} d x d y\right)$,

$$
\left(x^{2} G_{b}\right)\left(x^{-2} L_{b} u\right)=\left(x^{2} G_{b}\right) f=u-R u \Longrightarrow u=x^{2} G_{b} f+R u=\alpha+\beta .
$$

The first term, $\alpha \in x^{2} H_{b}^{2} \subset x^{2} \mathcal{L}^{2}\left(x^{n-1} d x d y\right)$. The second term $\beta \in \mathcal{L}^{2}$ $\left(x^{n-1} d x d y\right)$ has a polyhomogeneous expansion as $x \rightarrow 0$,

$$
\beta \sim \sum_{j=0}^{\infty} \sum_{k=0}^{N_{j}} x^{\gamma_{j}+k} \varphi_{j}(y) .
$$

Above $\gamma_{j}$ is an indicial root for the operator $L_{b}$ and $\varphi_{j}$ is an eigensection for the induced geometric Laplacian on $(Y, h)$. Then,

$$
u=\alpha+\sum_{j=0}^{\infty} \sum_{k=0}^{N_{j}} x^{\gamma_{j}+k} \varphi_{j}(y)
$$

where $\alpha \in x^{2} \mathcal{L}^{2}\left(x^{n-1} d x d y\right)$. This decomposition plays a key role in our proof of spectral convergence.

\subsection{Friedrichs domain of the conic Laplacian}

A geometric Laplacian $\Delta_{0}$ on a conic manifold is an unbounded operator on $\mathcal{L}^{2}$ sections of the bundle. It can be extended to various domains in $\mathcal{L}^{2}$. 
The minimal domain $\mathcal{D}_{\text {min }}$ is the completion of $\mathcal{C}_{c}^{\infty}\left(M_{0}\right)$ with respect to the norm $\|u\|+\left\|\Delta_{0} u\right\|$, while the maximal domain

$$
\mathcal{D}_{\max }=\left\{u \in \mathcal{L}^{2}\left(M_{0}\right) \mid \Delta_{0} u \in \mathcal{L}^{2}\left(M_{0}\right)\right\}
$$

Both $\mathcal{D}_{\text {min }}$ and $\mathcal{D}_{\text {max }}$ are dense in $\mathcal{L}^{2}\left(M_{0}\right)$, and the extension of the Laplacian to either domain is a closed operator. On complete manifolds $\mathcal{D}_{\text {min }}=\mathcal{D}_{\text {max }}$ by the Gaffney-Stokes theorem [14]. Conic metrics are incomplete and these domains will not in general be equal. The Friedrichs domain $\mathcal{D}_{\mathrm{F}}$ lies between $\mathcal{D}_{\text {min }}$ and $\mathcal{D}_{\text {max }}$ and is the closure of the graph of $\Delta_{0}$ in $\mathcal{L}^{2}$ with respect to the densely defined Hermitian form,

$$
Q(u, v)=\int_{M_{0}}\langle\nabla u, \nabla v\rangle .
$$

The extension of the Laplacian to the Friedrichs domain, known as the Friedrichs extension of the Laplacian, preserves the operator's lower bound and is essentially self adjoint. Here, we work exclusively with the Friedrichs extension of the conic Laplacian.

For elements of $\mathcal{D}_{\text {max }}$, with $u \in \mathcal{L}^{2}$ and $\Delta_{0} u=f \in \mathcal{L}^{2}$, we have the expansion (4.1) from the preceding section,

$$
u=\alpha+\sum_{j=0}^{\infty} \sum_{k=0}^{N_{j}} x^{\gamma_{j}+k} \varphi_{j}(y)
$$

The volume form on $M_{0}$ near the singularity is asymptotic to $x^{n-1} d x d y$. Therefore, the exponents $\gamma_{j}$ must all be strictly greater than $-\frac{n}{2}$. For $v \in$ $\mathcal{D}_{\text {min }} \subset \mathcal{D}_{\text {max }}$ the decomposition (4.1) and the definition of $\mathcal{D}_{\text {min }}$ imply that $\mathcal{D}_{\text {min }} \subset x^{2} \mathcal{L}^{2}$. The equality of $\mathcal{D}_{\text {min }}$ and $\mathcal{D}_{\text {max }}$ then depends on the indicial roots of $L_{b}=x^{2} \Delta_{0}$. For further discussion of domains of the conic Laplacian, see [16], whose results include:

$$
\begin{aligned}
\mathcal{D}_{\mathrm{F}}=\{f & \in \mathcal{L}^{2}: \Delta_{0} f \in \mathcal{L}^{2} \text { and } \\
& \left.\quad f=\mathcal{O}\left(x^{(2-n+\delta) / 2}\right) \text { as } x \rightarrow 0, \text { for some } \delta>0\right\} .
\end{aligned}
$$

We will use this characterization of the domain of the Friedrichs extension of the conic Laplacian in the proof of the first theorem. 


\section{Spectral convergence}

We now have all the necessary ingredients to prove spectral convergence.

Theorem 5.1. Let $\left(M_{0}, g_{0}\right)$ be a compact Riemannian n-manifold with isolated conic singularity, and let $\left(Z, g_{z}\right)$ be an ac space, with $n \geq 3$. Assume $\left(M, g_{\epsilon}\right)$ converges asymptotically conically to $\left(M_{0}, g_{0}\right)$. Let $\left(E_{0}, \nabla_{0}\right)$ and $\left(E_{z}, \nabla_{z}\right)$ be rank $k$ Hermitian vector bundles over $\left(M_{0}, g_{0}\right)$ and $\left(Z, g_{z}\right)$, respectively, so that each of these bundles in a neighborhood of the boundary is the pullback from a bundle over the boundary $(Y, h)$. Let $\Delta_{0}, \Delta_{z}$ be the corresponding Friedrichs extensions of geometric Laplacians, and let $\Delta_{\epsilon}$ be the induced geometric Laplacian on $\left(M, g_{\epsilon}\right)$. Assume $\Delta_{z}$ has no $\mathcal{L}^{2}$ nullspace. Then the accumulation points of the spectrum of $\Delta_{\epsilon}$ as $\epsilon \rightarrow 0$ are precisely the points of the spectrum of $\Delta_{0}$, counting multiplicity.

The theorem follows from the inclusion accumulation $\sigma\left(\Delta_{\epsilon}\right) \subset \sigma\left(\Delta_{0}\right)$, the reverse inclusion accumulation $\sigma\left(\Delta_{\epsilon}\right) \supset \sigma\left(\Delta_{0}\right)$, and correct multiplicities.

\subsection{Accumulation $\sigma\left(\Delta_{\epsilon}\right) \subset \sigma\left(\Delta_{0}\right)$}

We extract a smoothly convergent sequence of eigensections corresponding to a converging sequence of eigenvalues as $\epsilon \rightarrow 0$ and show that the limit section of this sequence is an eigensection for the conic metric, and its eigenvalue is the accumulation point. For this argument, we work with sequences of metrics $\left\{g_{\epsilon_{j}}\right\}$ which we abbreviate $\left\{g_{j}\right\}$ with corresponding Laplacians $\Delta_{j}$.

Let $\lambda\left(\epsilon_{j}\right)$ be an eigenvalue of $\Delta_{j}$, with eigensection $f_{j}$. Assume that $\lambda\left(\epsilon_{j}\right) \rightarrow \bar{\lambda}$. Over any compact set $K \subset M_{0}^{0}, g_{j}=g_{\epsilon_{j}}$ converges smoothly to $g_{0}$ by Lemma 2.6, thus so do the coefficients of $\Delta_{j}$. Hence, normalizing $f_{j}$ by $\sup _{M}\left|f_{j}\right|=1$, it follows using standard elliptic estimates and the ArzelaAscoli theorem that $f_{j}$ converges in $\mathcal{C}^{\infty}$ on any compact subset of $M_{0}^{0}$ [22]. Furthermore, the limit section $\bar{f}$ satisfies the limiting equation

$$
\Delta_{0} \bar{f}=\bar{\lambda} \bar{f}
$$

However, we do not yet know that $\bar{f} \not \equiv 0$, nor, even if this limit is nontrivial, that it lies in the domain of the Friedrichs extension of $\Delta_{0}$. This is the content of the arguments to follow.

5.1.1. Weight functions Let $\phi_{\epsilon}: M_{0, \epsilon}-M_{0, \delta} \rightarrow Z_{1 / \epsilon}-Z_{1 / \delta}$ as in Definition 2.3. We identify $Z_{1 / \delta}$ with a fixed $K \subset U \subset M$ so that $M_{0, \epsilon}-M_{0, \delta} \cong$ $(U-K), K \cong Z_{1 / \delta}$. 
Let

$$
w_{\epsilon}= \begin{cases}c & \text { on } M-U, \\ \epsilon\left(\phi_{\epsilon}^{-1}\right)^{*} \rho & \text { on } U-K, \\ c \epsilon & \text { on } K .\end{cases}
$$

Above, $c$ is a constant, and no generality is lost by assuming $c=1$. Let $w_{j}=w_{\epsilon_{j}}$. For some $\delta>0$ to be chosen later, replacing $f_{j}$ by $\frac{f_{j}}{\left\|w_{j}^{\delta} f_{j}\right\|_{\infty}}$ we assume the supremum of $\left|f_{j} w_{j}^{\delta}\right|$ is 1 on $M$. Since $M$ is compact, $\left|f_{j}\right|$ attains a maximum at some point $p_{j} \in M$, and we may assume $p_{j}$ converges to some $\bar{p} \in M$. The argument splits into three cases depending on how and where $p_{j}$ accumulates in $M$.

5.1.2. Case 1: $w_{j}\left(p_{j}\right) \rightarrow c>0$ as $j \rightarrow \infty$. In this case, the points $\left\{p_{j}\right\}$ accumulate in a compact subset of $M-U$, which we may identify with a compact subset of $M_{0}^{0}$ by Lemma 2.6. So, we may assume that these points converge to some point $\bar{p} \neq p$ (the conic singular point). The maximum of $\left|f_{j} w_{j}^{\delta}\right|$ on $M$ is 1 and occurs at $p_{j}$, so

$$
\left|f_{j}\right| \leq w_{j}^{-\delta} \text { on } M \text { for each } j \Longrightarrow\left|f_{j}\left(p_{j}\right)\right| \rightarrow c^{-\delta} \text { as } j \rightarrow \infty .
$$

The locally uniform $\mathcal{C}^{\infty}$ convergence of $f_{j}$ to $\bar{f}$ implies that $|\bar{f}|$ satisfies a similar bound,

$$
|\bar{f}| \leq x^{-\delta} \quad \text { as } x \rightarrow 0,
$$

and clearly $|\bar{f}(\bar{p})|=c^{-\delta} \neq 0$. By the dimension assumption $n \geq 3$ and the characterization of the Friedrichs domain of the Laplacian, we may choose $\delta$ so that

$$
\frac{2-n}{2}<-\delta<0
$$

Then $\bar{f}$ lies in the Friedrichs domain of the Laplacian and satisfies

$$
\Delta_{0} \bar{f}=\bar{\lambda} \bar{f}
$$

so $\bar{\lambda}$ is an eigenvalue of $\Delta_{0}$.

5.1.3. Case 2: $\left|w_{j}\left(p_{j}\right)\right| \leq c \epsilon_{j}$ as $j \rightarrow \infty$. Analysis on $Z$ in this case leads to a contradiction. Let $\phi_{j}=\phi_{\epsilon_{j}}$ and $\tilde{f}_{j}=f_{j}\left(\phi_{j}^{-1}\right)$. Let $\tilde{p}_{j}=\phi_{j}\left(p_{j}\right)$. Because $\left|f_{j} w_{j}^{\delta}\right|$ attains its maximum value of 1 at $p_{j},\left|\tilde{f}_{j}\left(\tilde{p}_{j}\right)\right|=\left(w_{j}\left(p_{j}\right)\right)^{-\delta}$. Rescale $f_{j}$ and $\tilde{f}_{j}$, replacing them respectively with $\left(w_{j}\left(p_{j}\right)\right)^{\delta} f_{j}$ and $\left(w_{j}\left(p_{j}\right)\right)^{\delta} \tilde{f}_{j}$, so that the maximum of $\left|\tilde{f}_{j} \rho^{\delta}\right|$ occurs at the point $\tilde{p}_{j} \in Z_{j}=Z_{1 / \epsilon_{j}}$ and is equal to 1 . Since $w_{j}\left(p_{j}\right)=\mathcal{O}\left(\epsilon_{j}\right), \rho\left(\tilde{p}_{j}\right)=\epsilon_{j}^{-1} w_{j}\left(p_{j}\right)$ stays bounded for all $j$, we assume $\tilde{p_{j}}$ converges to $\tilde{p} \in Z$. By Lemma 2.6, $\left(Z_{j},\left.\epsilon_{j}^{-2} \phi_{j}^{*} g_{j}\right|_{U}\right)$ converges 
smoothly to $\left(Z_{j}, g_{z}\right)$. This implies the following equation is satisfied by $\tilde{f}_{j}$ on $Z_{j}$,

$$
\Delta_{z} \tilde{f}_{j}=\epsilon_{j}^{2} \lambda\left(\epsilon_{j}\right) \tilde{f}_{j}+O\left(\epsilon_{j}\right)
$$

Since $\lambda\left(\epsilon_{j}\right)$ converge to $\bar{\lambda}$ and $\left|\tilde{f}_{j} \rho^{\delta}\right| \leq 1$ on $Z_{j}$,

$$
\Delta_{z} \tilde{f}_{j} \rightarrow 0 \text { as } j \rightarrow \infty \text {, on any compact subset of } Z \text {. }
$$

This implies $f_{j} \rightarrow \bar{f}$ on $M$ and correspondingly, $\tilde{f}_{j} \rightarrow \tilde{f}$ locally uniformly $\mathcal{C}^{\infty}$ on $Z$, and $\tilde{f}$ satisfies

$$
\Delta_{z} \tilde{f}=0, \quad\left|\tilde{f} \rho^{\delta}\right| \leq 1
$$

Equality holds in the second equation at the point $p$. This shows that $\tilde{f}$ is not identically zero on $Z$ and $\tilde{f}=O\left(\rho^{-\delta}\right)$ as $\rho \rightarrow \infty$. Since $\tilde{f}$ is smooth on any compact subset of $Z$ and is therefore in $\mathcal{L}_{\text {loc }}^{2}(Z)$, choosing $\delta>n-2$ contradicts the assumption that $Z$ has trivial $\mathcal{L}^{2}$ nullspace.

5.1.4. Case 3: $w_{j}\left(p_{j}\right) \rightarrow 0, \frac{\epsilon_{j}}{w_{j}\left(p_{j}\right)} \rightarrow 0$ as $j \rightarrow \infty$. In this case, the points $\phi_{j}\left(p_{j}\right) \rightarrow \infty$ in $Z$, so we rescale and derive a contradiction on the complete cone over $(Y, h)$. Consider the coordinates $(\rho, y)$ on $Z$ defined for $\rho \geq \rho_{1}$. In these coordinates $g_{z}=d \rho^{2}+\rho^{2} h(\rho)$. Let $r_{j}=\frac{\epsilon_{j}}{w_{j}\left(p_{j}\right)} \rho$ and $\tilde{g}_{j}$ on $Z_{j}$ be defined by

$$
\tilde{g_{j}}=\left(\frac{\epsilon_{j}}{w_{j}\left(p_{j}\right)}\right)^{2} g_{z}
$$

Then,

$$
\left(Z_{j}, \tilde{g}_{j}\right) \cong\left(\left(\frac{\rho_{1} \epsilon_{j}}{w_{j}\left(p_{j}\right)}, \frac{1}{w_{j}\left(p_{j}\right)}\right)_{r_{j}} \times Y, d r_{j}^{2}+r_{j}^{2} h\left(\frac{r_{j} \epsilon_{j}}{w_{j}\left(p_{j}\right)}\right)\right)
$$

As $j \rightarrow \infty, h\left(\frac{r_{j} \epsilon_{j}}{w_{j}\left(p_{j}\right)}\right)$ converges smoothly to $h$, and

$$
\tilde{g}_{j} \rightarrow g_{C}=d r^{2}+r^{2} h
$$

on the complete cone $C$ over $(Y, h)$. Let $\tilde{f}_{j}=\left(w_{j}\left(p_{j}\right)\right)^{\delta} f_{j}\left(\phi_{j}^{-1}\right)$. Since $\left|f_{j} w_{j}^{\delta}\right|$ $\leq 1$ with equality at $p_{j}$,

$$
\left|\tilde{f}_{j} r_{j}^{\delta}\right| \leq 1 \text { on }\left(Z_{j}, \tilde{g}_{j}\right) \text { with equality at } \tilde{p}_{j}=\phi_{j}\left(p_{j}\right) \text {. }
$$


Let $\tilde{\Delta}_{j}$ on $Z_{j}$ be the Laplacian induced by $\tilde{g}_{j}$ on $Z_{j}$,

$$
\tilde{\Delta}_{j}=\frac{w_{j}\left(p_{j}\right)^{2}}{\epsilon_{j}^{2}} \Delta_{Z}
$$

Then,

$$
\tilde{\Delta}_{j} \tilde{f}_{j}=\epsilon_{j}^{2} \frac{w_{j}\left(p_{j}\right)^{2}}{\epsilon_{j}^{2}} \lambda\left(\epsilon_{j}\right) \tilde{f}_{j}+O\left(\epsilon_{j}\right) \text { on }\left(Z_{j}, \tilde{g}_{j}\right)
$$

Since $w_{j}\left(p_{j}\right) \rightarrow 0$ as $j \rightarrow \infty$, there is a locally uniform $\mathcal{C}^{\infty} \operatorname{limit} f_{c}$ of $\left\{\tilde{f}_{j}\right\}$ on $C$ which satisfies

$$
\left|f_{c} r^{\delta}\right| \leq 1, \quad \Delta_{c} f_{c}=0
$$

Since the points $\tilde{p_{j}}$ stay at a bounded radial distance with respect to the radial variable $r_{j}$ on $Z_{j}$, we may assume $\tilde{p}_{j} \rightarrow p_{c}$ for some $p_{c} \in C$. At this point, $\left|f_{c}\left(p_{c}\right) r\left(p_{c}\right)^{\delta}\right|=1$, so $f_{c}$ is not identically zero. By separation of variables (see, for example, [27]), $f_{c}$ has an expansion in an orthonormal eigenbasis $\left\{\phi_{j}\right\}$ of $\mathcal{L}^{2}(Y, h)$,

$$
f_{c}=\sum_{j \geq 0} a_{j,+} r^{\gamma_{j,+}} \phi_{j}(y)+a_{j,-} r^{\gamma_{j,-}} \phi_{j}(y),
$$

where $\gamma_{j,+/-}$ are indicial roots corresponding to $\phi_{j}$ and $a_{j,+/-} \in \mathbb{C}$. In order for $\left|f_{c} r^{\delta}\right| \leq 1$ globally on $C$, we must have only one term in this expansion; $f_{c}=a_{j} r^{-\delta} \phi_{j}(y)$. Because the indicial roots are discrete, we may choose $\delta$ so that $-\delta$ is not an indicial root. This is a contradiction.

\section{2. $\sigma\left(\Delta_{0}\right) \subset$ accumulation $\sigma\left(\Delta_{\epsilon}\right)$}

We use the Rayleigh-Ritz characterization of the eigenvalues [4]. Let $\lambda_{l}\left(\epsilon_{j}\right)$ be the $l$ th eigenvalue of $\Delta_{j}$ and let

$$
R_{j}(f):=\frac{\langle\nabla f, \nabla f\rangle_{j}}{\langle f, f\rangle_{j}}
$$

The subscript $j$ indicates that the inner product is taken with respect to the $\mathcal{L}^{2}$ norm on $M$ with the $g_{j}$ metric. The eigenvalues are characterized using Mini-Max by

$$
\lambda_{l}\left(\epsilon_{j}\right)=\inf _{\operatorname{dim} L=l, L \subset \mathcal{C}^{1}(M)} \sup _{f \in L, f \neq 0} R_{j}(f) .
$$


Similarly, this characterization holds for the eigenvalues of the Friedrichs extension of the conic Laplacian which are known to be discrete (see [6], for example). Because $\mathcal{C}_{0}^{\infty}\left(M_{0}\right)$ is dense in $\mathcal{L}^{2}\left(M_{0}\right)$, we may restrict to subspaces contained in $\mathcal{C}_{0}^{\infty}\left(M_{0}\right)$. Then, the $l$ th eigenvalue of $\Delta_{0}$ is

$$
\bar{\lambda}_{l}=\inf _{\operatorname{dim} L=l, L \subset \mathcal{C}_{0}^{\infty}\left(M_{0}\right)} \sup _{f \in L, f \neq 0} R_{0}(f) .
$$

Let $\bar{\lambda}_{l}$ be the $l$ th eigenvalue in the spectrum of $\Delta_{0}$. Fix $\epsilon>0$. Then there exists $L \subset \mathcal{C}_{0}^{\infty}$ with $\operatorname{dim}(L)=l$ and

$$
\sup _{f \in L, f \neq 0} R_{0}(f)<\bar{\lambda}_{l}+\epsilon
$$

Since any $f \in L$ is also in $\mathcal{C}_{0}^{\infty}(M)$ and because $L$ is finite dimensional, by the local convergence of $g_{j}$ to $g_{0}$, for large $j$

$$
\left|R_{j}(f)-R_{0}(f)\right|<\epsilon \text {, for any } f \in L .
$$

Since $\lambda_{l}\left(\epsilon_{j}\right)$ is the infimum

$$
\lambda_{l}\left(\epsilon_{j}\right) \leq \bar{\lambda}_{l}+2 \epsilon
$$

This shows $\left\{\lambda_{l}\left(\epsilon_{j}\right)\right\}$ is bounded in $j$, and so we extract a convergent subsequence and a corresponding convergent sequence of eigensections which exists by our previous arguments. For each $l$, we take

$$
\begin{gathered}
\lambda_{l}\left(\epsilon_{j}\right) \rightarrow \mu_{l} \leq \bar{\lambda}_{l}, \\
f_{j, l} \rightarrow u_{l}, \quad \Delta_{0} u_{l}=\mu_{l} u_{l} .
\end{gathered}
$$

These limit eigensections $u_{l}$ are seen to be orthogonal as follows. Fix $l, k$, with $f_{j, k} \rightarrow u_{k}$ and $f_{j, l} \rightarrow u_{l}$. Since $\mathcal{C}_{0}^{\infty}\left(M_{0}\right)$ is dense in $\mathcal{L}^{2}\left(M_{0}\right)$, we may choose a smooth cutoff function $\chi$ vanishing identically near the singularity in $M_{0}$ such that

$$
\begin{gathered}
\left\|\chi u_{k}-u_{k}\right\|_{L^{2}\left(M_{0}\right)}<\epsilon, \\
\left\|\chi u_{l}-u_{l}\right\|_{L^{2}\left(M_{0}\right)}<\epsilon, \\
\operatorname{Vol}_{j}(M-\operatorname{spt}(\chi))<\epsilon .
\end{gathered}
$$


Then on the support of $\chi, g_{j} \rightarrow g_{0}$ uniformly so for large $j$,

$$
\begin{gathered}
\left|\left\langle u_{k}, u_{l}\right\rangle_{0}-\left\langle\chi u_{k}, \chi u_{l}\right\rangle_{0}\right|<\epsilon, \\
\left|\left\langle\chi u_{k}, \chi u_{l}\right\rangle_{0}-\left\langle\chi u_{k}, \chi u_{l}\right\rangle_{j}\right|<\epsilon, \\
\left|\left\langle\chi u_{k}, \chi u_{l}\right\rangle_{j}-\left\langle\chi u_{k}, \chi f_{j, l}\right\rangle_{j}\right|<\epsilon, \\
\left|\left\langle\chi u_{k}, \chi f_{j, l}\right\rangle_{j}-\left\langle\chi f_{j, k}, \chi f_{j, l}\right\rangle_{j}\right|<\epsilon .
\end{gathered}
$$

Since the eigensections for $\Delta_{j}$ were chosen to be orthonormal and the volume of $(M-\operatorname{support}(\chi))$ is small with respect to $g_{j}$,

$$
\left|\left\langle\chi f_{j, k}, \chi f_{j, l}\right\rangle_{j}\right|<2 \epsilon
$$

Thus, $\left\langle u_{k}, u_{l}\right\rangle_{0}$ can be made arbitrarily small and $u_{k}, u_{l}$ are orthogonal for $l \neq k$. We complete this basis to form an eigenbasis of $\mathcal{L}^{2}\left(M_{0}\right)$. Let $\bar{f}_{l}$ be an arbitrary element of this eigenbasis with eigenvalue $\bar{\lambda}_{l}$. We wish to show that this $\bar{f}_{l}$ is actually the $u_{l}$ above, defined to be the limit of (a subsequence of) $\left\{f_{j, l}\right\}$, and hence the corresponding $\mu_{l}$ is equal to $\bar{\lambda}_{l}$. Again, assume the smooth cut-off function $\chi$ is chosen so that

$$
\left\|\chi \bar{f}_{l}-\bar{f}_{l}\right\|_{L^{2}\left(M_{0}\right)}<\epsilon
$$

For each $j$, we expand $\chi \bar{f}_{l}$ in eigensections of $\Delta_{j}$,

$$
\chi \bar{f}_{l}=\sum_{k=0}^{\infty} a_{j, k} f_{j, k}, \quad \text { where } a_{j, k}=\left\langle\chi \bar{f}_{l}, f_{j, k}\right\rangle_{j}
$$

Now, fix $k$ and choose $\chi$ such that

$$
\left\|\chi u_{k}-u_{k}\right\|_{L^{2}\left(M_{0}\right)}<\epsilon .
$$

Then,

$$
\begin{gathered}
\left|\left\langle\chi \bar{f}_{l}, f_{j, k}\right\rangle_{0}-\left\langle\chi \bar{f}_{l}, f_{j, k}\right\rangle_{j}\right|<\epsilon, \\
\left|\left\langle\chi \bar{f}_{l}, f_{j, k}\right\rangle_{j}-\left\langle\chi \bar{f}_{l}, u_{k}\right\rangle_{j}\right|<\epsilon, \\
\mid\left\langle\left\langle\chi \bar{f}_{l}, u_{k}\right\rangle_{j}-\left\langle\chi \bar{f}_{l}, u_{k}\right\rangle_{0}\right|<\epsilon, \\
\left|\left\langle\chi \bar{f}_{l}, u_{k}\right\rangle_{0}-\left\langle\bar{f}_{l}, u_{k}\right\rangle_{0}\right|<\epsilon .
\end{gathered}
$$

By the orthogonality $\left\langle\bar{f}_{l}, u_{k}\right\rangle_{0}=0$ if $\bar{f}_{l} \neq u_{k}$, and otherwise is 1 , so for each $k$, $a_{j, k} \rightarrow 0$ as $j \rightarrow \infty$, for all $k$ with $u_{k} \neq \bar{f}_{l}$. Because $\bar{f}_{l}$ is not identically zero, 
there must be some $k$ with $u_{k}=\bar{f}_{l}$. This shows that every eigensection of $\Delta_{0}$ is the limit of (a subsequence of) $\left\{f_{j, k}\right\}$, and the corresponding eigenvalue $\overline{\lambda_{k}}$ is the limit of the corresponding eigenvalues.

\subsection{Correct multiplicities}

We show by induction that the eigenvalues of $\Delta_{0}$ are achieved as accumulation points with correct multiplicity. The spectrum of $\Delta_{0}$ listed with multiplicity is

$$
\lambda_{1} \leq \lambda_{2} \leq \cdots, \quad \text { where } \Delta_{0} f_{i}=\lambda_{i} f_{i},
$$

and the eigensections are $\mathcal{L}^{2}$ orthonormal. For each $\epsilon$, the spectrum of $\Delta_{\epsilon}$ is

$$
\lambda_{1, \epsilon} \leq \lambda_{2, \epsilon} \leq \cdots, \quad \text { where } \Delta_{\epsilon} f_{i, \epsilon}=\lambda_{i, \epsilon} f_{i, \epsilon} .
$$

By previous arguments, there is a sequence $\left\{f_{1, j}\right\}$ which converges to $h_{1}$ and $\lambda_{1, j} \rightarrow \lambda_{1}$. If there is some other $\left\{f_{1, k}\right\} \rightarrow h_{2}$ where $h_{2}$ is also an eigensection for $\Delta_{0}$ with eigenvalue $\lambda_{1}$, then we have shown in preceding arguments that both $h_{1}$ and $h_{2}$ are equal to eigensections of $\Delta_{0}$ listed above, and both have eigenvalue $\lambda_{1}$. If the multiplicity of $\lambda_{1}$ is one, we must have $h_{1}=h_{2}=f_{1}$. By preceding arguments, the limit of any converging sequence $\lambda_{k, j}$ is $\lambda_{k}$, so that when $\lambda_{1}$ is an eigenvalue of multiplicity one, $\lambda_{1}<\lambda_{2}$, so $\lambda_{1}$ is an accumulation point of multiplicity one. If the multiplicity of $\lambda_{1}$ is greater than one, then $\lambda_{2}=\lambda_{1}$, and we have shown that there is a sequence $\lambda_{2, j} \rightarrow \lambda_{2}$, and we have shown that the corresponding limit eigensection $f_{2}$ is orthogonal to $f_{1}$. By these arguments and the preceding orthogonality arguments, it is clear that the multiplicity of $\lambda_{1}$ as an eigenvalue is equal to the multiplicity of $\lambda_{1}$ as an accumulation point. By induction, the same argument shows that all eigenvalues of $\Delta_{0}$ are achieved as accumulation points with the correct multiplicity, thereby completing the proof of spectral convergence.

\section{Heat kernels}

The heat kernels for each of the geometries in ac convergence are elements of a pseudodifferential heat operator calculus that is defined on a corresponding heat space. For the details in the construction of these heat calculi, kernels and spaces, see [37]. 


\section{1. $b$-Heat kernel}

Let $(M, g)$ be a $b$-manifold with local coordinates $z=(x, y)$ in a neighborhood of $\partial M$ so that near the boundary

$$
g=\frac{d x^{2}}{x^{2}}+h(x, y)
$$

Let $\left(z, z^{\prime}\right)$ be coordinates on $M \times M$ and let $\Delta_{b}$ be a geometric Laplacian on $M$. The $b$-heat kernel $H\left(z, z^{\prime}, t\right)$ is the Schwartz kernel of the fundamental solution of the heat operator $\partial_{t}+\Delta_{b}$. The heat kernel is a distributional section acting on smooth sections of $M$ and satisfying

$$
\begin{gathered}
\left(\partial_{t}+\Delta_{b}\right) H\left(z, z^{\prime}, t\right)=0, \quad t>0 \\
\left.H\right|_{t=0}=\delta\left(z-z^{\prime}\right) .
\end{gathered}
$$

By self adjointness since we work with the Friedrichs extension of $\Delta_{b}$,

$$
H\left(z, z^{\prime}, t\right)=H\left(z^{\prime}, z, t\right)^{*}
$$

For a smooth section $u$ on $M$,

$$
u(z, t):=\int_{M}\left\langle u\left(z^{\prime}\right), H\left(z, z^{\prime}, t\right)\right\rangle d z^{\prime}
$$

satisfies

$$
\left(\partial_{t}+\Delta_{b}\right) u(z, t)=0 \text { for } t>0, \quad u(z, 0)=u(z)
$$

Physically, $u(z, t)$ describes the heat on $M$ at time $t>0$ where the initial heat applied to $M$ is given by $u(z)$.

Recall the Euclidean heat kernel,

$$
G\left(z, z^{\prime}, t\right)=(4 \pi t)^{-n / 2} \exp \left(-\frac{\left|z-z^{\prime}\right|^{2}}{2 t}\right)
$$

For a compact manifold without boundary, the heat kernel can be constructed locally using the Euclidean heat kernel, Riemannian normal coordinates, the "transport equations" determined by the local geometry and Duhamel's principle; see for example [36]. On the interior of a manifold with boundary (or singularity), the Euclidean heat kernel is also a good model; however, near the boundary (or singularity) a different construction is required. 
6.1.1. $b$-Heat space It is convenient to study the heat kernel on a manifold with boundary (or singularity) as an element of a heat operator calculus defined on a corresponding heat space (Figure 2). This space is a manifold with corners constructed from $M \times M \times \mathbb{R}^{+}$by blowing up along submanifolds at which the heat kernel may have interesting or singular behavior. ${ }^{6}$ For example, the diagonal is always blown up at $t=0$, since away from the boundary the heat kernel behaves like the Euclidean heat kernel which is singular along the diagonal at $t=0$. To construct the $b$-heat space, we first blow up the codimension 2 corner at the boundary in both copies of $M$ to create the $b$-double space,

$$
M_{b}^{2}=[M \times M ; \partial M \times \partial M]
$$

The $b$-heat space $M_{b, h}^{2}$ is then

$$
M_{b, h}^{2}=\left[M_{b}^{2} \times \mathbb{R}_{t}^{+} ; \Delta(M \times M) \times\{t=0\}, d t\right],
$$

where $\Delta(M \times M)$ is the diagonal in $M \times M$. The $b$-heat space has five boundary faces, two of which result from blowing up. The remaining three boundary faces are at $t=0$ off the diagonal and at the boundary in each copy of $M$. More precisely, we have the following. ${ }^{7}$

\begin{tabular}{lll}
\hline Face & Geometry of face & Defining function in local coordinates \\
\hline$F_{110}$ & $N^{+}(Y \times Y) \times \mathbb{R}^{+}$ & $\rho_{110}=\left(x^{2}+\left(x^{\prime}\right)^{2}\right)^{1 / 2}$ \\
$F_{d 2}$ & $P N_{t}^{+}(\Delta(M \times M))$ & $\rho_{d 2}=\left(\left|z-z^{\prime}\right|^{4}+t^{2}\right)^{1 / 4}$ \\
$F_{100}$ & $Y \times(M-\partial M) \times \mathbb{R}^{+}$ & $\rho_{100}=x$ \\
$F_{010}$ & $Y \times(M-\partial M) \times \mathbb{R}^{+}$ & $\rho_{010}=x^{\prime}$ \\
$F_{001}$ & $(M-\partial M)^{2}-\Delta(M \times M)$ & $\rho_{001}=t$ \\
\hline
\end{tabular}

Above $P N_{t}^{+}$denotes the inward-pointing $t$ parabolic normal bundle, while $N^{+}$denotes the inward-pointing spherical normal bundle. Note that

\footnotetext{
${ }^{6}$ The key idea is to blow-up appropriate submanifolds and introduce a new "symbol" or "normal operator" for each blown-up face and then solve these normal operators. In the classical microlocal sense, we invert the symbol for each face. In this way, the complexity of the operator on the original space is handled geometrically by blowing up, thereby allowing one to use the powerful tools of classical microlocal analysis in non-classical (non-compact) geometric settings. For a detailed exposition, see [33].

${ }^{7}$ The subscript " $d$ " indicates a face created by blowing up along the diagonal, so for example $F_{d 2}$ is the face created by blowing up along the diagonal parabolically in the $t$ direction.
} 


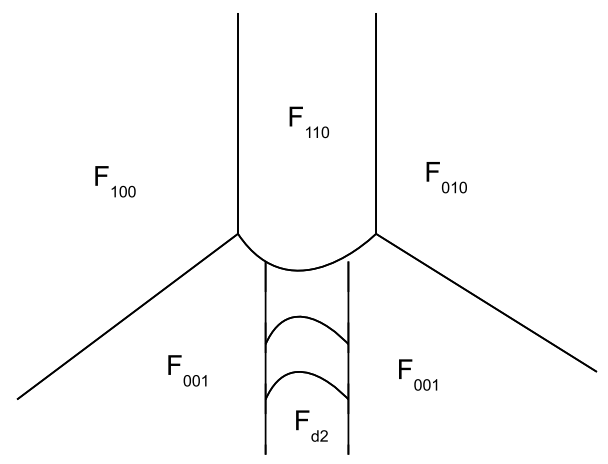

Figure 2: Schematic diagram of the $b$-heat space.

the local coordinates $x, x^{\prime}, t$ lift from $M \times M \times \mathbb{R}^{+}$to $M_{b, h}^{2}$ as follows:

$$
\beta^{*}(x)=\rho_{110} \rho_{100}, \quad \beta^{*}\left(x^{\prime}\right)=\rho_{110} \rho_{010}, \quad \beta^{*}(t)=\left(\rho_{d 2}\right)^{2} \rho_{001},
$$

so these coordinates are only local defining functions.

6.1.2. $b$-Heat calculus The $b$-heat calculus consists of distributional section half-density kernels on $M_{+}^{2}=M \times M \times \mathbb{R}^{+}$which are smooth on the interior and lift to be polyhomogeneous on $M_{b, h}^{2}$ with specified leading orders at the boundary faces. By constructing the $b$-heat kernel as an element of the $b$-heat calculus, it is polyhomogenous on $M_{b, h}^{2}$ with specified leading orders. Once the calculus is defined and the composition rule is proven, construction of the heat kernel as an element of the heat calculus proceeds like the classical microlocal constructions in $[19,20,38]$. The following definition is from [33].

Definition 6.1. For any $k \in \mathbb{R}$ and index set $E_{110}, A$ is an element of the $b$-heat calculus $\Psi_{b, H}^{E_{110}, k}$ if $A$ is smooth on the interior of $M_{b, h}^{2}$ and satisfies the following.

1. $A \in \mathcal{A}_{p h g}^{-1 / 2+E_{110}}\left(F_{110}\right)$.

2. $A$ vanishes to infinite order at $F_{001}, F_{100}$ and $F_{010}$.

3. $A \in \rho_{d 2}^{-((n+3) / 2)-k} \mathcal{C}^{\infty}\left(F_{d 2}\right)$.

Because the heat calculus is defined with half-densities, the normalizing factors at $F_{110}$ and $F_{d 2}$ simplify the composition rule. An element $A$ of the $b$-heat calculus is the Schwartz kernel of an operator acting on a smooth 
half-density section $f$ of $M$ by

$$
A f(z, t)=\int_{M}\left\langle A\left(z, z^{\prime}, t\right), f\left(z^{\prime}\right)\right\rangle d z^{\prime} .
$$

Furthermore, $A$ acts by convolution in the $t$ variable so for a smooth halfdensity section $f$ of $M \times \mathbb{R}_{t}^{+}$,

$$
A f(z, t)=\int_{0}^{t} \int_{M}\left\langle A\left(z, z^{\prime}, t-s\right), f\left(z^{\prime}, s\right)\right\rangle d z^{\prime} d s .
$$

Two elements of the $b$-heat calculus compose as follows.

Technical Theorem 6.2. Let $A \in \Psi_{b, H}^{k_{a}, \mathcal{A}}$ and let $B \in \Psi_{b, H}^{k_{b}, \mathcal{B}}$. Then the composition, $A \circ B$, is an element of $\Psi_{b, H}^{k_{a}+k_{b}, \mathcal{A} \cup \mathcal{B}}$.

The proof of this composition rule is in $[33] .{ }^{8}$

6.1.3. Construction of the $b$-heat kernel First we construct a model heat kernel $H_{1}$ as an element of the $b$-heat calculus that solves the heat equation up to an error vanishing to positive order at the boundary faces of $M_{b, h}^{2}$. On the interior of $M_{b, h}^{2}$ restricting to a coordinate patch with coordinates $\left(z, z^{\prime}, t\right)$, we locally define

$$
H_{1}\left(z, z^{\prime}, t\right):=(4 \pi t)^{-n / 2} e^{\left(\left|z-z^{\prime}\right|_{g}\right)^{2} / 2 t},
$$

where $\left|z-z^{\prime}\right|_{g}$ is the distance from $z$ to $z^{\prime}$ with respect to the metric $g$. As $t \rightarrow 0$ away from the diagonal, this construction immediately implies infinite order vanishing at $F_{001}$. At $F_{d 2}$ we solve exactly: for each $p \in M$ and for each point $z \in F_{d 2}$ in the fiber over $(p, p, 0)$, the heat kernel at that point is determined by the coefficients of the metric (and its derivatives) at $p$.

The normal operator of $\partial_{t}+\Delta_{b}$ is the restriction to $F_{110}$ of the lift of $\partial_{t}+\Delta_{b}$ to $M_{b, h}^{2}$. $H_{1}$ is defined at $F_{110}$ to be the kernel of a first-order parametrix of this normal operator and is smooth at this face. At $F_{100}, F_{010}$

\footnotetext{
${ }^{8}$ Note that in order for the composition to be defined, $A$ and $B$ must satisfy certain compatibility conditions. In all our applications, these conditions are a priori satisfied. The notation $\bar{U}$ denotes the extended union of index sets, see [28].
} 
and $F_{001}$, the model kernel vanishes to infinite order. As constructed, $H_{1}$ satisfies

$$
\left(\partial_{t}+\Delta\right) H_{1}=K_{1}, \quad H_{1} \in \Psi_{b, H}^{-2,0}
$$

$K_{1}$ vanishes to positive order at the boundary faces of $M_{b, h}^{2}$. Next, define

$$
H_{2}=H_{1}-H_{1} * K_{1},
$$

where now the error term,

$$
K_{2}=\left(\partial_{t}+\Delta\right) H_{2}
$$

vanishes to one order higher on each of the boundary faces of $M_{b, h}^{2}$ by the composition rule. This construction is iterated and the Borel summation [38] gives $H_{\infty} \in \Psi_{b, H}^{-2,0}$ with $H_{\infty}-H_{N}=O\left(t^{N-(n+3) / 2}\right)$, for $N>0$, so that

$$
\left(\partial_{t}+\Delta\right) H_{\infty}=K
$$

$K$ vanishes to infinite order on the boundary faces of $M_{b, h}^{2}$, so we may push $K$ forward to $M \times M \times \mathbb{R}^{+}$. We solve away the residual error term using the action of elements of the $b$-heat calculus as $t$-convolution operators. As a $t$-convolution operator, the heat kernel is the identity. Above, $K$ as a $t$-convolution operator is of the form $K=\mathrm{Id}-A$, where $A$ is a Volterra operator, and Id is the identity. An operator of this form has an inverse of the same form, so defining

$$
H:=H_{\infty}(\operatorname{Id}-A)^{-1}
$$

solves away this residual error term. By construction, the leading order behavior of the $b$-heat kernel is that of the model heat kernel and is summarized below.

\begin{tabular}{ll}
\hline Face & Leading order \\
\hline$F_{110}$ & $0 ; O\left(t^{-1}\right)$ as $t \rightarrow \infty$ \\
$F_{d 2}$ & $(-(n+3) / 2-(-2))$ \\
$F_{100}$ & $\infty$ order vanishing \\
$F_{010}$ & $\infty$ order vanishing \\
$F_{001}$ & $\infty$ order vanishing \\
\hline
\end{tabular}

\subsection{Conic heat kernel}

Let $\left(M_{0}, g_{0}\right)$ be a compact manifold with isolated conic singularity, and let $\Delta_{0}$ be the Friedrichs extension of a geometric Laplacian associated to a 
Hermitian vector bundle over $\left(M_{0}, g_{0}\right)$. We construct the conic heat kernel using the same steps for constructing the $b$-heat kernel: first we construct the conic heat space with its calculus and composition rule, then we solve the normal operators by constructing a model parametrix and use the calculus to solve up to infinite order, and finally we solve away the residual error using the action as a $t$-convolution operator.

6.2.1. The conic heat space This construction comes from [34]. The conic heat space $M_{0, h}^{2}$ is a manifold with corners obtained from $M_{0} \times M_{0} \times$ $\mathbb{R}^{+}=M_{0,+}^{2}$ by blowing up along two submanifolds (Figure 3 ),

$$
\begin{aligned}
& M_{0, h}^{2}:= {\left[\left[M_{0} \times M_{0} \times \mathbb{R}^{+} ; \partial M_{0} \times \partial M_{0} \times\{t=0\}, d t\right] ;\right.} \\
&\left.\Delta\left(M_{0}^{0} \times M_{0}^{0}\right) \times\{t=0\}, d t\right] .
\end{aligned}
$$

The conic heat space has five boundary faces described in the following table in which $z=(x, y)$ and $z^{\prime}=\left(x^{\prime}, y^{\prime}\right)$ are local coordinates in a neighborhood of the singularity in each copy of $M_{0}$ so that $x=0, x^{\prime}=0$ define the singularity as well as the boundary of $M_{0}$.

\begin{tabular}{lll}
\hline Face & Geometry of face & Defining function in local coordinates \\
\hline$F_{112}$ & $P N_{t}^{+}(Y \times Y)$ & $\rho_{112}=\left(x^{4}+\left(x^{\prime}\right)^{4}+t^{2}\right)^{1 / 4}$ \\
$F_{d 2}$ & $P N_{t}^{+}\left(\Delta\left(M_{0}^{0} \times M_{0}^{0}\right)\right)$ & $\rho_{d 2}=\left(\left|z-z^{\prime}\right|^{4}+t^{2}\right)^{1 / 4}$ \\
$F_{100}$ & $Y \times \mathbb{R}^{+}$ & $\rho_{100}=x$ \\
$F_{010}$ & $\mathbb{R}^{+} \times Y$ & $\rho_{010}=x^{\prime}$ \\
$F_{001}$ & $M_{0}^{0} \times M_{0}^{0}-\Delta\left(M_{0}^{0} \times M_{0}^{0}\right)$ & $\rho_{001}=t$ \\
\hline
\end{tabular}

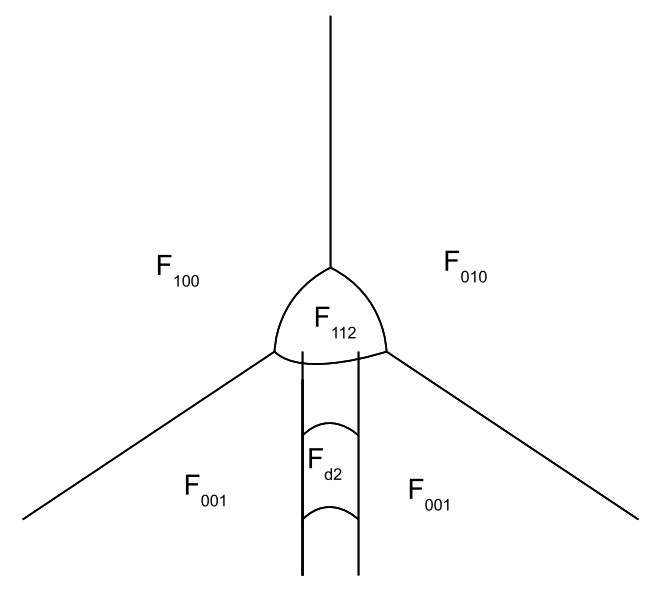

Figure 3: Schematic diagram of the conic heat space. 
Note that the coordinates $x, x^{\prime}, t$ lift from $M_{0} \times M_{0} \times \mathbb{R}^{+}$to $M_{0, h}^{2}$ as follows:

$$
\beta^{*}(x)=\rho_{100} \rho_{112}, \quad \beta^{*}\left(x^{\prime}\right)=\rho_{010} \rho_{112}, \quad \beta^{*}(t)=\rho_{112}^{2} \rho_{d 2}^{2} \rho_{001},
$$

so again these are only local defining functions.

6.2.2. The conic heat calculus Let $\mu$ be a conic half-density on $M_{0,+}^{2}$. We may assume

$$
\mu=\left(x x^{\prime}\right)^{(n-1) / 2} \sqrt{d z d z^{\prime} d t}=\sqrt{d V_{c} d t} .
$$

Fix also a smooth, non-vanishing half-density $\nu$ on $M_{0, h}^{2}$. Elements of the conic heat calculus are distributional section half densities on $M_{0,+}^{2}$ which are smooth on the interior and lift to be polyhomogeneous on $M_{0, h}^{2}$.

Definition 6.3. Let $k \in \mathbb{R}$ and let $E_{100}, E_{010}$ and $E_{112}$ be index sets. Then $A \in \Psi_{0, H}^{k, E_{100}, E_{010}, E_{112}}$ if $A$ is a smooth half-density section on the interior of $M_{0, h}^{2}$ and satisfies the following.

1. $A \in \mathcal{A}_{p h g}^{E_{100}}$ at $F_{100}$.

2. $A \in \mathcal{A}_{p h g}^{E_{010}}$ at $F_{010}$.

3. $A \in \mathcal{A}_{p h g}^{E_{112}}$ at $F_{112}$.

4. $A$ vanishes to infinite order at $F_{001}$.

5. $A \in \rho_{d 2}^{(-(n+3) / 2)-k} \mathcal{C}^{\infty}\left(F_{d 2}\right)$.

With this normalization, the conic heat kernel has order $k=-2$, and the composition rule is the following.

Technical Theorem 6.4. Let $A \in \Psi_{0, h}^{A_{100}, A_{010}, A_{112}, k_{a}}$ and $B \in \Psi_{0, H}^{B_{100}, B_{010}, B_{112}, k_{b}}$ with leading index terms satisfying

$\beta_{112}+\alpha_{010}>0, \quad \alpha_{112}+\beta_{100}>0, \quad-k_{a}>0, \quad-k_{b}>0, \quad \beta_{100}+\alpha_{010}>-1$.

Then, the composition $B \circ A$ is an element of $\Psi_{0, H}^{A_{100}, B_{010}, \Gamma_{112}, k}$ with $\Gamma_{112}=$ $A_{112} \cup B_{112}$ and $k=\left(k_{a}+k_{b}\right)$.

The proof of this theorem is in [37] and is originally due to [34]; see also $[15,27]$. 
Away from $F_{112}, F_{100}$ and $F_{010}$, the model heat kernel is locally constructed using the Euclidean heat kernel. At the front face $F_{112}$, which arises from blowing up the singularity, the coordinates $x, x^{\prime}, t$ vanish. Instead, consider the projective coordinates ${ }^{9}$

$$
s=\frac{x}{x^{\prime}}, \quad s^{\prime}=x^{\prime}, \quad \tau=\frac{t}{\left(x^{\prime}\right)^{2}}
$$

In these coordinates, the heat operator

$$
\partial_{t}+\Delta=\left(s^{\prime}\right)^{-2}\left(\partial_{\tau}+\left(\partial_{s}\right)^{2}+s^{-2} \Delta_{h}\right)
$$

where $\Delta_{h}$ is the induced geometric Laplacian on the boundary $(Y, h)$. Since $s^{\prime}$ is a defining function for $F_{112}$, we see that the normal operator is the heat operator for the exact cone over $(Y, h)$, so we define the model heat kernel at $F_{112}$ to be

$$
H_{0}\left(s, s^{\prime}, y, y^{\prime}, \tau\right)
$$

where $H_{0}$ is the heat kernel for the exact cone over $(Y, h)$. Using the scaling properties of the heat kernel for an exact cone, this is equivalent to

$$
\left(\rho_{112}\right)^{-n} H_{0}\left(s, 1, y, y^{\prime}, \tau\right)
$$

which is well defined away from $F_{010}$ and has a polyhomogeneous expansion up to $F_{100}$; see $[5,23]$. With the Friedrichs extension, symmetry in space variables defines the model heat kernel on all of $F_{112}$, and we define it on $F_{100}$ and $F_{010}$ by extending the polyhomogeneous expansion at the corners of these faces with $F_{112}$.

\subsection{Asymptotically conic scattering heat kernel}

A summary of the ac scattering heat kernel, space and calculus is given here; details are contained in the appendix. Let $\left(\bar{Z}, g_{z}\right)$ be an ac scattering metric with boundary defined by $\{x=0\}$ and with local coordinates $z=(x, y)$ near the boundary. Let $\Delta_{z}$ be the Friedrichs extension of a geometric Laplacian on $\bar{Z}$.

\footnotetext{
${ }^{9}$ It is generally helpful to work with projective coordinates since differential operators transform nicely under projective coordinate changes.
} 
6.3.1. Asymptotically conic scattering heat space First, we construct the ac scattering double space,

$$
\bar{Z}_{\mathrm{sc}}^{2}:=\left[[\bar{Z} \times \bar{Z} ; \partial \bar{Z} \times \partial \bar{Z}] ; \Delta(Y \times Y) \cap F_{110}\right],
$$

where $F_{110}$ is the face created by the first blowup. This construction comes from $[17$, Figure 4]. The ac scattering heat space is

$$
\bar{Z}_{s c, h}^{2}=\left[\bar{Z}_{s c}^{2} \times \mathbb{R}^{+} ; \Delta(Z \times Z) \times\{t=0\}, d t\right] .
$$

The ac scattering heat space has six boundary faces described in the following table.

\begin{tabular}{lll}
\hline Face & Geometry of face & Defining function in local coordinates \\
\hline$F_{220}$ & $N^{+}(\Delta(Y \times Y)) \times \mathbb{R}^{+}$ & $\rho_{220}=\left(x^{2}+\left(x^{\prime}\right)^{2}+\left|y-y^{\prime}\right|^{2}\right)^{1 / 2}$ \\
$F_{110}$ & $N^{+}((Y \times Y)$ & \\
& $-\Delta(Y \times Y)) \times \mathbb{R}^{+}$ & $\rho_{110}=\left(x^{2}+\left(x^{\prime}\right)^{2}\right)^{1 / 2}$ \\
$F_{100}$ & $Z \times Y \times \mathbb{R}^{+}$ & $\rho_{100}=x$ \\
$F_{010}$ & $Y \times Z \times \mathbb{R}^{+}$ & $\rho_{010}=x^{\prime}$ \\
$F_{d 2}$ & $P N_{t}^{+}(\Delta(Z \times Z))$ & $\rho_{d 2}=\left(\left|z-z^{\prime}\right|^{4}+t^{2}\right)^{1 / 4}$ \\
$F_{001}$ & $(Z \times Z)-\Delta(Z \times Z)$ & $\rho_{001}=t$ \\
\hline
\end{tabular}

6.3.2. Asymptotically conic scattering heat calculus Elements of the ac scattering heat calculus are distributional section half- densities of $\bar{Z}_{+}^{2}$ which are smooth on the interior and lift to be polyhomogeneous on $\bar{Z}_{s c, h}^{2}$. Let $\mu$ be a smooth, non-vanishing half- density on $\bar{Z}_{+}^{2}$, and let $\nu$ be a smooth, non-vanishing half- density on $\bar{Z}_{\mathrm{sc}, h}^{2}$.

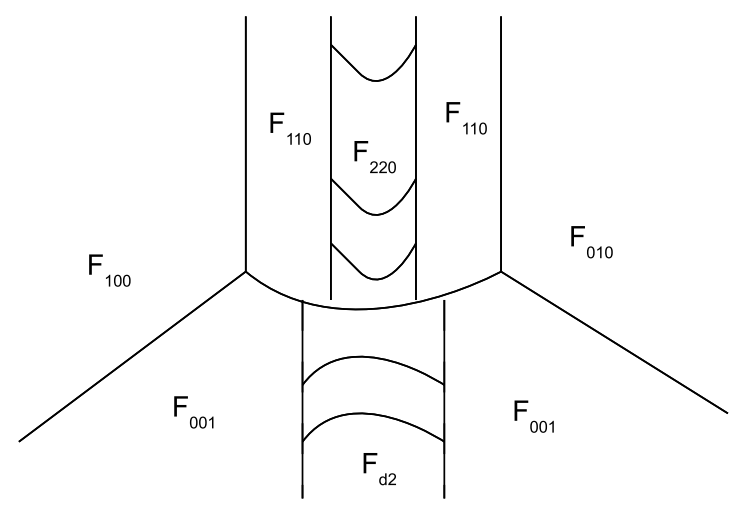

Figure 4: Schematic diagram of the ac scattering heat space. 
Definition 6.5. For any $k \in \mathbb{R}$ and index sets $E_{110}$ and $E_{220}, A \in \Psi_{\mathrm{sc}, H}^{E_{110}, E_{220}, k}$ if $A$ is smooth on the interior of $\bar{Z}_{\mathrm{sc}, h}^{2}$ and satisfies the following.

1. $A \in \mathcal{A}_{p h g}^{-(1 / 2)+E_{110}}$ at $F_{110}$.

2. $A \in \mathcal{A}_{p h g}^{-((n+2) / 2)+E_{220}}$ at $F_{220}$.

3. $A$ vanishes to infinite order at $F_{001}, F_{100}$ and $F_{010}$.

4. $A \in \rho_{d 2}^{-((n+3) / 2)-k} \mathcal{C}^{\infty}\left(F_{d 2}\right)$.

Two elements of the ac scattering heat calculus compose as follows.

Technical Theorem 6.6. Let $A \in \Psi_{\mathrm{sc}, H}^{A_{110}, A_{220}, k_{a}}$, and $B \in \Psi_{\mathrm{sc}, H}^{B_{110}, B_{220}, k_{b}}$.

Then, the composition $B \circ A$ is an element of $\Psi_{\mathrm{sc}, H}^{A_{110} \cup B_{110}, A_{220} \cup B_{220}, k_{a}+k_{b}}$.

The ac scattering heat kernel is constructed analogously to the $b$ and conic heat kernels. The model heat kernel in this case is the lift of the Euclidean heat kernel to $\bar{Z}_{\mathrm{sc}, h}^{2} \cdot{ }^{10}$

Technical Theorem 6.7. Let $\left(\bar{Z}, g_{z}\right)$ be an ac scattering metric with boundary $(Y, h)$. Let $(E, \nabla)$ be a Hermitian vector bundle over $\left(Z, g_{z}\right)$ which induces a compatible bundle over $(Y, h)$. Let $\Delta$ be a geometric Laplacian on $\left(\bar{Z}, g_{z}\right)$ associated to the bundle $(E, \nabla)$. Then there exists $H \in \Psi_{s c, H}^{E_{110}, E_{220},-2}$ satisfying

$$
\begin{gathered}
\left(\partial_{t}+\Delta\right) H\left(z, z^{\prime}, t\right)=0, \quad t>0 \\
H\left(z, z^{\prime}, 0\right)=\delta\left(z-z^{\prime}\right) .
\end{gathered}
$$

Moreover, $H$ vanishes to infinite order at $F_{110}$ and is smooth up to $F_{220}$.

The proof of this theorem is in the appendix.

\section{Heat kernel convergence}

These heat kernels interact on the acc heat space.

\footnotetext{
${ }^{10}$ Since the standard example of an ac scattering metric is the radial compactification of $\mathbb{R}^{n}$, it is natural that the ac scattering model heat kernel is the lift of the Euclidean heat kernel to $\bar{Z}_{\mathrm{sc}, h}^{2}$.
} 


\subsection{Asymptotically conic convergence heat space}

This construction is similar to the heat space constructions in Section 6 and the acc double space construction in Section 3. First, let

$$
\mathcal{H}_{0}:=\left\{\epsilon=\epsilon^{\prime}\right\} \subset \mathcal{S} \times \mathcal{S}
$$

Next, let

$$
\mathcal{H}_{1}:=\left[\mathcal{H}_{0} \times \mathbb{R}_{t}^{+} ; Y \times Y \times\{t=0\}, d t\right] .
$$

This blowup must be done first to create the $F_{112}$ face in the conic heat space. The scalar variables on $\mathcal{S} \times \mathcal{S} \times \mathbb{R}_{t}^{+}$are $\left(x, r, x^{\prime}, r^{\prime}, t\right),{ }^{11}$ so by our notation, the face created by this blowup is $F_{1111,2}$. Let

$$
\mathcal{H}_{2}:=\left[\mathcal{H}_{1} ; Z \times Z \times\{t=0\}, d t\right] .
$$

This blowup is less obvious: the ac metric is scaled by $\epsilon^{2}$, so the corresponding Laplacian is scaled by $\epsilon^{-2}$, and the time should also be scaled. To create a face with rescaled time, we perform this blowup, and the resulting face is $F_{1010,2}$, which has the geometry of a compactified $b$-heat space at $t, \epsilon=0$.

Finally, the acc heat space results from blowing up the closure of the lift of the diagonal in $\mathcal{S} \times \mathcal{S}$ at $\{t=0\}$,

$$
\mathcal{H}:=\left[\mathcal{H}_{2} ; \overline{\beta^{*}(\Delta(\mathcal{S} \times \mathcal{S}-(Y \times Y)) \times\{t=0\})}, d t\right] .
$$

The face created by this last blowup is $F_{d 2}$.

The $\epsilon=0$ boundary faces of $\mathcal{H}$ are summarized below:

\begin{tabular}{lll}
\hline $\mathcal{S} \times \mathcal{S} \times \mathbb{R}_{t}^{+}$corner & $\mathcal{H}$ face & geometry \\
\hline$x=0, x^{\prime}=0, r=0$, & & \\
$r^{\prime}=0, t=0$ & $F_{1111,2}$ & $P N^{+}(Y \times Y)$ \\
$x=0, x^{\prime}=0, t=0$, & $F_{1010,2}$ & {$\left[\bar{Z} \times \bar{Z} \times \mathbb{R}_{\tau}^{+} ; Y \times Y\right] ;$} \\
& & $\Delta(Z \times Z) \times\{0\}, d \tau]$ \\
$x=0, x^{\prime}=0$, & $F_{1010}$ & {$\left[Z \times Z \times \mathbb{R}^{+}-\{t=0\}\right]$} \\
$r=0, r^{\prime}=0$ & $F_{0101}$ & $M_{0, h}^{2}$ \\
$x=0, r^{\prime}=0$ & $F_{1001}$ & {$\left[\bar{Z} \times M_{0} \times \mathbb{R}^{+} ; Y \times Y \times\{0\}, d t\right]$} \\
$r=0, x^{\prime}=0$ & $F_{0110}$ & {$\left[M_{0} \times \bar{Z} \times \mathbb{R}^{+} ; Y \times Y \times\{0\}, d t\right]$} \\
$\Delta(\mathcal{S} \times \mathcal{S}) \times\{t=0\}$ & $F_{d 2}$ & $P N^{+}(\Delta(\mathcal{S} \times \mathcal{S}))$ \\
$\{t=0\}$ & $F_{0000,1}$ & $\left(\left\{\epsilon=\epsilon^{\prime}\right\} \subset \mathcal{S} \times \mathcal{S}\right)-\Delta(\mathcal{S} \times \mathcal{S})$ \\
\hline
\end{tabular}

${ }^{11}$ Note that these variables are not independent; they are related by $x r=x^{\prime} r^{\prime}=\epsilon$. 


\subsubsection{Asymptotically conic convergence half-density calculations} We calculate the lift to $\mathcal{H}$ of $d V g_{\epsilon} d V g_{\epsilon}^{\prime} d t d \epsilon$. The Jacobian determinant factors which result from blowing up are

$$
\left(\rho_{1111,2}\right)^{4}\left(\rho_{1010,2}\right)^{2}\left(\rho_{d 2}\right)^{n+1} \text {. }
$$

Next, we calculate the lift of the variables $x, x^{\prime}, r, r^{\prime}$ to $\mathcal{H},{ }^{12}$

$$
\begin{aligned}
\beta^{*}(x) & =\rho_{1010} \rho_{1010,2} \rho_{1111,2} \rho_{1001}, \\
\beta^{*}\left(x^{\prime}\right) & =\rho_{1010} \rho_{1010,2} \rho_{1111,2} \rho_{0110}, \\
\beta^{*}(r) & =\rho_{1111,2} \rho_{0101} \rho_{0110}, \\
\beta^{*}\left(r^{\prime}\right) & =\rho_{1111,2} \rho_{0101} \rho_{1001} .
\end{aligned}
$$

We calculate the volume form $d V g_{\epsilon}$ in a neighborhood of the faces of $\mathcal{H}$ at $\epsilon=0$. At $F_{0101}, d V g_{\epsilon} \sim d V_{0}$ and

$$
\begin{aligned}
d V g_{\epsilon} d V g_{\epsilon}^{\prime} \sim & \left(\rho_{1111,2} \rho_{1010} \rho_{1010,2}\right)^{2 n-2}\left(\rho_{1001} \rho_{0110}\right)^{n-1} \\
& \times\left(\rho_{1111.2}\right)^{4}\left(\rho_{1010,2}\right)^{2}\left(\rho_{d 2}\right)^{n+1} \mu
\end{aligned}
$$

At $F_{1010}$ and $F_{1010,2}, d V g_{\epsilon} \sim \epsilon^{2} d V_{z}$, consequently

$$
\begin{aligned}
d V g_{\epsilon} d V g_{\epsilon}^{\prime} \sim & \left(\rho_{1111,2}\right)^{2 n-2}\left(\rho_{1010,2} \rho_{1010}\right)^{2 n}\left(\rho_{0101}\right)^{-2}\left(\rho_{1001} \rho_{0110}\right)^{n-1} \\
& \times\left(\rho_{1111.2}\right)^{4}\left(\rho_{1010,2}\right)^{2}\left(\rho_{d 2}\right)^{n+1} \mu
\end{aligned}
$$

where $\mu$ is a smooth non-vanishing spatial density on $\mathcal{H} .^{13}$

Then, we arrive at the following half-density calculation at $F_{0101}$ and $F_{1111,2}$,

$$
\begin{aligned}
\beta^{*}\left(\sqrt{d V g_{\epsilon} d V g_{\epsilon}^{\prime} d t d \epsilon}\right) \sim & \left(\rho_{1111,2}\right)^{n+1}\left(\rho_{1010,2}\right)^{n}\left(\rho_{1010}\right)^{n-1} \\
& \times\left(\rho_{1001} \rho_{0110}\right)^{(n-1) / 2}\left(\rho_{d 2}\right)^{(n+1) / 2} \sqrt{\nu}
\end{aligned}
$$

where $\nu$ is a smooth non-vanishing density on $\mathcal{H}$. At $F_{1010}$ and $F_{1010,2}$,

$$
\begin{aligned}
\beta^{*}\left(\sqrt{d V g_{\epsilon} d V g_{\epsilon}^{\prime} d t d \epsilon}\right) \sim & \left(\rho_{1111,2}\right)^{n+1}\left(\rho_{1010,2}\right)^{n+1}\left(\rho_{1010}\right)^{n}\left(\rho_{0101}\right)^{-1} \\
& \times\left(\rho_{1001} \rho_{0110}\right)^{(n-1) / 2}\left(\rho_{d 2}\right)^{(n+1) / 2} \sqrt{\nu}
\end{aligned}
$$

${ }^{12}$ The recipe for these exponents is: (codimension of space variables -1) + (codimension of parabolic variables $* 2$ ).

${ }^{13}$ We have used that $d V_{0} \sim x^{n-1} d x d y$ and $d V_{z} \sim \frac{\epsilon^{n}}{r^{n+1}} d r d y$ and that $\epsilon=x r=x^{\prime} r^{\prime}$. 


\subsection{Asymptotically conic convergence heat calculus}

The acc heat calculus is a parameter $(\epsilon)$ dependent operator calculus incorporating the smooth, conic, and $b$-heat calculi. ${ }^{14}$

Definition 7.1. The acc heat calculus of order $k$, written $\Psi_{\text {acc, } H}^{k, E_{0101}, E_{1010,2}, E_{111,2}}$, consists of half-density kernels $A$ which are smooth on the interior of $\mathcal{H}$ and satisfy the following.

1. For each $\epsilon>0, A$ restricts to an element of $\Psi_{\epsilon, H}^{k}$, the standard heat calculus of order $k$ for $\left(M, g_{\epsilon}\right){ }^{15}$

2. In a neighborhood of $F_{1010,2}, A$ has an asymptotic expansion in $\rho_{1010,2}$ with index set $E_{1010,2}$ and coefficients in the $b$-heat calculus of order $k$. Such an expansion is of the form

$$
A \sim \sum_{j \geq 1} \sum_{0 \leq p_{0} \leq p \leq p_{j}}\left(\rho_{1010,2}\right)^{\alpha_{j}}\left(\log \rho_{1010,2}\right)^{p} A_{j, l}
$$

with $A_{j, l} \in \Psi_{b, H}^{k, E_{110}^{j}}$. Above, if for some $j, p_{j}=0$, then there are no log terms.

3. In a neighborhood of $F_{0101}, A$ has an asymptotic expansion in $\rho_{0101}$ with index set $E_{0101}$, and coefficients are elements of the conic heat calculus of order $k$.

4. In a neighborhood of $F_{1111,2}, A$ has an asymptotic expansion in $\rho_{1111,2}$ with index set $E_{1111,2}$ so that the coefficients in the conic heat calculus are of order $k$ for the exact cone over $Y$.

5. $A$ has a smooth polyhomogeneous expansion up to the side faces $F_{1001}, F_{1010}, F_{0110}$, and these expansions extend smoothly to the corners meeting the other faces of $\mathcal{H}$.

${ }^{14}$ The ac scattering heat calculus was expected to arise in the acc heat space and calculus, but after calculating the normal operators as $\epsilon \rightarrow 0$, it became clear that the $b$-heat space and calculus rather than the ac scattering heat space and calculus were needed.

${ }^{15}$ The heat space for a smooth compact manifold $M$ is $M_{h}^{2}:=[M \times M \times$ $\left.\mathbb{R}_{t}^{+} ; \Delta(M) \times\{t=0\}, d t\right]$. The standard heat calculus $\Psi_{H}^{k}$ consists of half-density sections which are smooth on the interior of $M_{h}^{2}$, vanish identically at $t=0$ away from the blown-up face, and which are $\rho^{-(1 / 2)(n+3)-k} \mathcal{C}^{\infty}$ at the blown-up face defined by $\rho$; see, for example, [33,37]. 
The composition rule is not required for the proof of our main theorem, but we would expect it to follow from the composition rules for the smooth (compact manifolds), $b$, and conic heat calculi, together with the result for combining polyhomogeneous index sets as in [28].

Theorem 7.2. Let $\left(M_{0}, g_{0}\right)$ be a compact Riemannian $n$-manifold with isolated conic singularity, and let $\left(Z, g_{z}\right)$ be an ac space, with $n \geq 2$. Assume $\left(M, g_{\epsilon}\right)$ converges asymptotically conically to $\left(M_{0}, g_{0}\right)$. Let $\left(E_{0}, \nabla_{0}\right)$ and $\left(E_{z}\right.$, $\left.\nabla_{z}\right)$ be rank $k$ Hermitian vector bundles over $\left(M_{0}, g_{0}\right)$ and $\left(Z, g_{z}\right)$, respectively, so that each of these bundles in a neighborhood of the boundary is the pullback from a bundle over the boundary $(Y, h)$. Let $\Delta_{0}$ and $\Delta_{z}$ be the corresponding Friedrichs extensions of geometric Laplacians, and let $\Delta_{\epsilon}$ be the induced geometric Laplacian on $\left(M, g_{\epsilon}\right)$. Then the associated heat kernels $H_{\epsilon}$ have a full polyhomogeneous expansion as $\epsilon \rightarrow 0$ on the acc heat space with the following leading terms:

- At the conic front face, $F_{0101}, H_{\epsilon}\left(z, z^{\prime}, t\right) \rightarrow H_{0}\left(z, z^{\prime}, t\right)$, the heat kernel for $\left(M_{0}, g_{0}\right)$ with half-density factor

$(*)=\left(\rho_{1111,2}\right)^{n+1}\left(\rho_{1010,2}\right)^{n}\left(\rho_{1010}\right)^{n-1}\left(\rho_{1001} \rho_{0110}\right)^{(n-1) / 2}\left(\rho_{d 2}\right)^{(n+1) / 2} \sqrt{\nu}$.

- At the rescaled $b$ front face, $F_{1010,2}, H_{\epsilon}\left(z, z^{\prime}, t\right) \rightarrow\left(\rho_{1010,2}\right)\left(\rho_{1010}\right)$ $\left(\rho_{0101}\right)^{-1} H_{b}(\tau)$, the $b$ heat kernel with rescaled time variable $\tau$ and with half-density $(*)$.

- At the exact conic front face, $F_{1111,2}, H_{\epsilon}\left(z, z^{\prime}, t\right) \rightarrow H_{0}(\widetilde{\tau})$, the heat kernel for the exact cone with rescaled time variable $\widetilde{\tau}$ with half-density $(*)$.

- At the side faces $F_{1001}, F_{0110}$ and the residual $b$ face $F_{1010}$, the heat kernel has polyhomogeneous expansion with leading term vanishing to at least second order.

This convergence is uniform in $\epsilon$ for bounded time and moreover, the error term is bounded by $C \epsilon t^{N}$ as $t \rightarrow 0$, for any $N \in \mathbb{N}_{0}$, where $C$ may depend on $N$.

\subsection{Proof}

This proof is modeled after the parametrix construction of [33]. First, we lift the operator $\partial_{t}+\Delta_{\epsilon}$ to $\mathcal{H}$ and construct the acc model heat kernel as an element of the acc heat calculus which solves the normal operators of $\partial_{t}+\Delta_{\epsilon}$ on $\mathcal{H}$. We use the $b$-heat calculus and introduce the acc conic triple 
heat space to use the conic heat calculus and solve up to infinite order, then we estimate the error term of this solution kernel and finally solve away the residual error.

7.3.1. Lifted heat operator We must carefully choose good coordinates in a neighborhood of each of the front faces $F_{0101}, F_{1010,2}$ and $F_{1111,2}$ and calculate the leading term of $\partial_{t}+\Delta_{\epsilon}$ as $\epsilon \rightarrow 0$, or in the terminology of $[1,28,33]$, we calculate the "normal operators" at the $\epsilon=0$ boundary faces of $\mathcal{H}$. In a neighborhood of $F_{0101}, g_{\epsilon}$ is smoothly approaching the conic metric, so

$$
\partial_{t}+\Delta_{\epsilon} \rightarrow \partial_{t}+\Delta_{0}
$$

In a neighborhood of $F_{1010,2}$, the metric $g_{\epsilon} \rightarrow \epsilon^{-2} g_{z}$, so

$$
\partial_{t}+\Delta_{\epsilon} \rightarrow \partial_{t}+\epsilon^{-2} \Delta_{z}=(x)^{-2}\left(\partial_{\tau}+\Delta_{b}\right),
$$

where $\tau=t /\left(x^{2}\right)$. In the above calculation, we have used $x r=\epsilon$ together with the relation between the ac scattering Laplacian and the rescaled $b$ Laplacian $\Delta_{z}=r^{2} \Delta_{b}$. Note that $x$ lifts to define $F_{1010}, F_{1010,2}$ and $F_{1111,2}$. This indicates that the leading part of $\partial_{t}+\Delta_{\epsilon}$ at $F_{1010,2}$ is

$$
\left(\rho_{1010,2} \rho_{1010} \rho_{1111,2}\right)^{-2}\left(\partial_{\tau}+\Delta_{b}\right) \text {. }
$$

In a neighborhood of $F_{1111,2}$, the scalar variables $\left(x, x^{\prime}, r, r^{\prime}, t\right)$ are not good coordinates since they all vanish. Better coordinates are the projective $\left(s, s^{\prime}, \sigma, \sigma^{\prime}, \tau\right)$, where

$$
s=\frac{x}{x^{\prime}}, \quad s^{\prime}=x^{\prime}, \quad \sigma=\frac{r}{x^{\prime}}, \quad \sigma^{\prime}=\frac{r^{\prime}}{x^{\prime}}, \quad \tilde{\tau}=\frac{t}{\left(x^{\prime}\right)^{2}} .
$$

Note that

$$
\left(r \partial_{r}\right)=\left(\sigma \partial_{\sigma}\right), \quad x r=\epsilon \Longrightarrow \frac{r^{2}}{\epsilon^{2}}=x^{-2}
$$

At $F_{1111,2}$ we see both the conic metric $g_{0}$ near the singularity and the rescaled ac scattering metric $\epsilon^{2} g_{z}$ near the boundary. Using the projective coordinates around the conic singularity, we compute

$$
\partial_{t}+\Delta_{\epsilon} \rightarrow\left(s^{\prime}\right)^{-2}\left(\partial_{\tilde{\tau}}+\left(\partial_{s}\right)^{2}+(s)^{-2}\left(\Delta_{h}\right)=\left(s s^{\prime}\right)^{-2}\left(\partial_{\tau}+\left(s \partial_{s}\right)^{2}+\Delta_{h}\right),\right.
$$

where $\Delta_{h}$ is the Laplacian on $Y$ for $h=h(x=0)$, and we note that $\tau=\frac{t}{\left(s s^{\prime}\right)^{2}}$. Using the projective coordinates near the boundary of $\bar{Z}$, we compute

$$
\partial_{t}+\Delta_{\epsilon} \rightarrow\left(s s^{\prime}\right)^{-2}\left(\partial_{\tau}+\left(\sigma \partial_{\sigma}\right)^{2}+\Delta_{h}\right)=\left(s^{\prime}\right)^{-2}\left(\partial_{\tilde{\tau}}+(s)^{-2}\left(\left(\sigma \partial_{\sigma}\right)^{2}+\Delta_{h}\right),\right.
$$


where in the last equality we have used $x r=\epsilon=x^{\prime} r^{\prime}$, which gives $s \partial_{s}=\sigma \partial_{\sigma}$. Since $\left(s s^{\prime}\right)=x$ lifts to $\mathcal{H}$ to define $F_{1111,2}, F_{1010}$ and $F_{1010,2}$ as does $s^{\prime}$, this indicates that the leading term of $\partial_{t}+\Delta_{\epsilon}$ at $F_{1111,2}$ is

$$
\left(\rho_{1111,2} \rho_{1010} \rho_{1010,2}\right)^{-2}\left(\partial_{\widetilde{\tau}}+\Delta_{0, s}\right),
$$

where $\Delta_{0, s}$ is the Laplacian for the exact cone over $(Y, h)$. Note that these calculations demonstrate that the model operators are consistent at the corners.

These calculations together with the half-density calculation indicate how to define the acc model heat kernel as a parametrix for $\partial_{t}+\Delta_{\epsilon}$ as $\epsilon \rightarrow 0$.

7.3.2. Asymptotically conic convergence model heat kernel, $H_{1}$

- At $F_{0101}$, let $H_{1}\left(z, z^{\prime}, t, \epsilon\right) \sim H_{0}$, the heat kernel for $\left(M_{0}, g_{0}\right)$, with halfdensity factor ${ }^{16}$

$(*)=\left(\rho_{1111,2}\right)^{n+1}\left(\rho_{1010,2}\right)^{n}\left(\rho_{1010}\right)^{n-1}\left(\rho_{1001} \rho_{0110}\right)^{(n-1) / 2}\left(\rho_{d 2}\right)^{(n+1) / 2} \sqrt{\nu}$.

- At $F_{0101,2}$, let $H_{1}\left(z, z^{\prime}, t, \epsilon\right) \sim\left(\rho_{1010} \rho_{1010,2}\right)\left(\rho_{0101}\right)^{-1} H_{b}(\tau)(*)$, where $H_{b}(\tau)$ is the $b$ heat kernel with rescaled time variable.

- At $F_{1111,2}$, let $H_{1}\left(z, z^{\prime}, t, \epsilon\right) \sim H_{0}(\widetilde{\tau})(*)$, where $H_{0}(\widetilde{\tau})$ is the heat kernel for the exact cone over $(Y, h)$ with rescaled time.

At the side faces of $\mathcal{H}$, it is instructive to recall the side faces in the conic heat space. The conic heat kernel has normal operator

$$
\partial_{t}+\left(\partial_{x}\right)^{2}+x^{-2} \Delta_{h}
$$

where $\Delta_{h}$ is the induced Laplacian on the boundary $(Y, h)$. At the side face $F_{100}, x$ vanishes and there is no natural "normal operator". However, the side faces meet the front face, so we define the conic model heat kernel on the side faces by extending the asymptotic expansion from the front face to the side faces and using symmetry of the Friedrichs extension. When the conic density is included, the conic model heat kernel vanishes to positive order at the side faces. Similarly, there is no natural normal operator at

\footnotetext{
${ }^{16}$ Note that we do not require any additional vanishing factors to account for the singular factors in the normal operators since the half-density already includes sufficient vanishing factors to remove the singular factors when the normal operators are applied.
} 
$F_{1010}, F_{1001}$ and $F_{0110}$. However, the acc model heat kernel is defined on $F_{0101}$ which meets $F_{1001}$ and $F_{0110}$, and so it may be defined on these faces by extending the expansion as in the conic case. At the corner of $F_{1010,2}$ with $F_{1010}$, the rescaled time variable $\tau \rightarrow \infty$, and the $b$ heat kernel vanishes to positive order as $t \rightarrow \infty$, so we define the acc model heat kernel at $F_{1010}$ by extending the expansion at $F_{1010,2}$ as $\tau \rightarrow \infty$. For $\epsilon>0$, let $H_{1}\left(z, z^{\prime}, t, \epsilon\right)$ be the heat kernel for $\partial_{t}+\Delta_{\epsilon}$.

\subsubsection{Asymptotically conic convergence model heat kernel con-} struction along diagonal at $t=0$ In a neighborhood of the faces diffeomorphic to the parabolic normal bundle of the diagonal, $P N^{+}(\Delta)$, we carry out a local construction as in [33, chapter 7]. Let $X$ be a manifold; since this construction is the same for $X=M, X=M_{0}$ and $X=\bar{Z}$, we use $X$ to simplify notation. Let $F_{X}$ denote the $P N^{+}(\Delta(X))$ face in $\mathcal{H}$, where $\Delta(X)$ is the diagonal in $X \times X$. Let $N\left(\partial_{t}+\Delta_{X}\right)$ be the restriction to $F_{X}$ of the lift of $\left(\partial_{t}+\Delta_{X}\right)$ to $\mathcal{H}$, where $\Delta_{X}$ is our geometric Laplacian on $X$. With the heat calculus normalization at $F_{X}$, an element $A$ of the acc heat calculus of order $-k$ restricts to $F_{X}$ as follows

$$
N(A)=\left.t^{(k+n+2) / 2} A\right|_{F_{X}}
$$

As in [33] we observe that $F_{X}$ is naturally diffeomorphic to a radial compactification of the tangent space of $X$, with each fiber of $F_{X}$ over $(x, x, 0)$ diffeomorphic to the tangent space at $x$. From [33, 7.15],

$$
N\left(t\left(\partial_{t}+\Delta_{X}\right) A\right)=\left[\sigma\left(\Delta_{X}\right)-\frac{1}{2}(R+n+k+2)\right] N(A),
$$

where $R$ is the radial vector field on the fibers of $T X$. Note that if $G_{0}$ satisfies

$$
t\left(\partial_{t}+\Delta_{X}\right) G_{0}=O\left(t^{\infty}\right) \text { as } t \rightarrow 0,\left.\quad G_{0}\right|_{t=0}=\delta\left(x-x^{\prime}\right)
$$

then $G_{0}$ also satisfies

$$
\left(\partial_{t}+\Delta_{X}\right) G_{0}=O\left(t^{\infty}\right) \text { as } t \rightarrow 0,\left.\quad G_{0}\right|_{t=0}=\delta\left(x-x^{\prime}\right)
$$

So, we may work with $t\left(\partial_{t}+\Delta_{X}\right)$ as in [33]. Our initial parametrix $G_{0}$ has order $k=-2$ at $F_{X}$. From (7.1), we have the following equation for $G_{0}$,

$$
\left[\sigma\left(\Delta_{X}\right)-\frac{1}{2}(R+n)\right] N\left(G_{0}\right)=0
$$


From $[33,7.13]$, in order for $G_{0}$ to satisfy the initial condition, it must satisfy

$$
\int_{\text {fiber }} N\left(G_{0}\right)=1
$$

Since these conditions are fiber-by-fiber, we introduce local coordinates so that

$$
\sigma\left(\Delta_{X}\right)=D_{1}^{2}+\cdots+D_{n}^{2} \text { on } T_{x} X
$$

Then we have

$$
\left[D_{1}^{2}+\cdots+D_{n}^{2}-\frac{1}{2}(R+n)\right] N\left(G_{0}\right)=0
$$

SO

$$
N\left(G_{0}\right)=(2 \pi)^{-n / 2} \exp \left(-\frac{|X|_{x}^{2}}{4}\right)
$$

is the desired solution, where $X$ is a projective local coordinate on $F_{x}$, $X=\frac{x-x^{\prime}}{t^{1 / 2}}($ see $[33,7.36])$, and $|*|_{x}$ is the Riemannian norm on $T X$ induced by the metric at $x$. To see that this is the desired solution, consider the Fourier transform of (7.3) with $u=\left.N\left(G_{0}\right)\right|_{T_{x} X}$,

$$
\left(\xi \partial_{\xi}+2|\xi|^{2}\right) \hat{u}=0, \quad \hat{u}(0)=1 .
$$

Then by standard results in ordinary differential equations, the expression in (7.4) is the unique decaying solution.

Now we may iterate this to solve up to higher order. Assume we have found $G_{0}, \ldots, G_{k}$ satisfying

$$
t\left(\partial_{t}+\Delta_{X}\right) G_{j}=R_{j}
$$

where $R_{j}$ is of order $-3-j$ at $F_{X}$. To find $G_{k+1}=G_{k}-T_{k}$, we wish to solve

$$
t\left(\partial_{t}+\Delta_{X}\right) T_{k}=R_{k}+R_{k+1}
$$

where we have already found $R_{k}$ of order $-3-k$, and $R_{k+1}$ will be of order $-4-k$. Lifting to $T X$ this becomes

$$
\left[\sigma\left(\Delta_{X}\right)-\frac{1}{2}(R+n-j-1)\right] N\left(T_{k}\right)=N\left(R_{k}\right),
$$


which we may again solve via Fourier transform. Letting $u=N\left(T_{k}\right)$ and $f=N\left(R_{k}\right)$, we find

$$
\hat{u}(\xi)=\int_{0}^{1} \exp \left((r-1)|\xi|^{2}\right) \hat{f}(r \xi) r^{k+1} d r
$$

is the desired solution. This completes the inductive construction for all $k$. Now the successive $T_{j}=G_{j+1}-G_{j}$ give a formal power series at $F_{X}$ which can be summed by Borel's lemma so that $G$ is order -2 at $F_{X}$, and satisfies (7.2). We then set the acc model heat kernel $H_{1}=G$ in a neighborhood of $F_{X}$.

7.3.4. Asymptotically conic convergence model heat kernel construction off diagonal at $\boldsymbol{t}=\mathbf{0}$ Consider $F_{1010,2} \cong \bar{Z}_{b, h, \tau}^{2}$. This face has the following geometry.

\begin{tabular}{lll}
\hline Boundary face & Geometry of face & Arising from \\
\hline$F_{b d 2}$ & $P N^{+}(\Delta(Z \times Z))$ & Parabolic blowup of diagonal \\
& $N^{+}(Y \times Y) \times \mathbb{R}^{+}$ & at $\tau=0$ \\
$F_{b 110}$ & $Y \times Z \times \mathbb{R}^{+}$ & Blowup of $Y \times Y$ for all $\tau$ \\
$F_{b 100}$ & $Z \times Y \times \mathbb{R}^{+}$ & Boundary in first copy of $Z$ \\
$F_{b 010}$ & $(Z \times Z)-\Delta(Z \times Z)$ & Boundary in second copy of $Z$ \\
$F_{b 001}$ & $\tau=0$ Away from diagonal \\
\hline
\end{tabular}

At this face, $H_{1}$ is asymptotic to $H_{b}\left(z, z^{\prime}, \tau\right)$, which vanishes to infinite order at the side faces $F_{b 100}$ and $F_{b 010}$. At the diagonal face $F_{b d 2}$, we have solved $H_{1}$ up to error vanishing to infinite order in $t$. So, we have at this point an approximation $H_{1}$ whose error vanishes to infinite order on the interior of $F_{1010,2}$ and at all boundary faces except $F_{b 110}$. The indicial operator for $\Delta_{b, \sigma}$ at $F_{b 110}$ is

$$
I\left(\Delta_{b, \sigma}\right)=\left(\sigma \partial_{\sigma}\right)^{2}+\Delta_{y}
$$

on $\mathbb{R}_{\sigma}^{+} \times Y$. We would like to find $u$ which is polyhomogeneous on $\bar{Z}_{b, h, \tau}^{2}$ and solves

$$
\left.\left(\partial_{\tau}+\Delta_{b}\right)\right|_{F_{b 110}} u=-\left.K\right|_{F_{b 110}}
$$

where $K\left(z, z^{\prime}, t, \epsilon\right)=\left(\partial_{t}+\Delta_{\epsilon}\right) H_{1}$. Since $H_{1}$ is polyhomogeneous on $\mathcal{H}$ and smooth up to $F_{b 110}$, so also the error term $K$. Expanding $K$, where we use 
simply $\rho$ for the projective defining function for $F_{b 110}$,

$$
\left.K\right|_{F_{b 110}} \sim \sum_{j \geq 0}(\rho)^{j} k_{j}, \quad k_{j} \in \mathcal{C}_{0}^{\infty} .
$$

Expanding the desired solution $u$,

$$
u \sim \sum_{j \geq 0}(\rho)^{j} u_{j}
$$

We may use either separation of variables expanding in eigenfunctions of $\Delta_{y}$ or the Mellin transform [33], to find $u_{0}$ satisfying

$$
\left(\partial_{\tau}+I\left(\Delta_{b}\right)\right) u_{0}=-k_{0},
$$

with $u_{0}$ vanishing to infinite order at the side faces $F_{b 100}$ and $F_{b 010}$. Since $\Delta_{b}-I\left(\Delta_{b}\right)=\rho L_{1}$, where $L_{1}$ is also a $b$-differential operator, we may now iteratively solve for $u_{1}, u_{2}, \ldots$, to solve the equation to increasingly higher order. Recall that in the projective coordinates near this face, $\sigma^{\prime}$ defines $F_{b 110}$, and since the operator does not differentiate with respect to $\sigma^{\prime}=r$, the defining function commutes past the operator. Using Borel summation, we construct $u$ so that

$$
\left(\partial_{\tau}+\Delta_{b}\right) u=-K+K_{2},
$$

where $K_{2}$ vanishes to infinite order at $F_{b 110}$. Using a smooth cutoff function $\chi$ supported in a neighborhood of these faces, the second approximation $H_{2}=H_{1}+\chi u$ now satisfies

$$
\left(\partial_{\tau}+\Delta_{b}\right) H_{2}=K_{2},
$$

where $K_{2}$ vanishes to infinite order on both the interior and all boundary faces of $F_{1010,2}$.

A similar exact construction applies to $F_{1111,2}$, since this face is the heat space for the exact cone over $(Y, h)$.

7.3.5. Asymptotically conic convergence conic triple heat space Since the error now vanishes to infinite order at all boundary faces except $F_{0101}$, we restrict attention to this face. It is convenient to use the conic heat calculus composition rule which requires the conic triple space. We construct a partial acc triple heat space,${ }^{17}$ the acc conic triple heat space,

${ }^{17}$ The full acc triple heat space is constructed in the second appendix although it is not needed for this proof. 
which contains the conic triple heat space, thereby allowing us to solve away the error at $F_{0101}$ using the conic composition rule. The acc conic triple heat space $\mathcal{H}_{c}^{3}$ is a submanifold constructed from $\mathcal{S}^{3} \times \mathbb{R}^{+} \times \mathbb{R}^{+}$by eight blowups. Let $X, X^{\prime}, X^{\prime \prime}$ denote the three copies of the submanifold $X$ in $X^{3}$. The blowups are listed in the following table by the order in which the blowups are performed together with the name of the face created.

\begin{tabular}{ll}
\hline Blowup & Face \\
\hline$Y \times Y^{\prime} \times Y^{\prime \prime} \times\left\{t=0, t^{\prime}=0\right\}, d t, d t^{\prime}$ & $F_{11122}$ \\
$Y \times Y^{\prime} \times\{t=0\}, d t$ & $F_{11020}$ \\
$Y^{\prime} \times Y^{\prime \prime} \times\left\{t^{\prime}=0\right\}, d t^{\prime}$ & $F_{01102}$ \\
$Y \times Y^{\prime \prime} \times\left\{t^{\prime \prime}=\left|t-t^{\prime}\right|=0\right\}, d t^{\prime \prime}$ & $F_{10122}$ \\
$\Delta\left(\mathcal{S} \times \mathcal{S}^{\prime} \times \mathcal{S}^{\prime \prime}\right) \times\left\{t=0, t^{\prime}=0\right\}, d t, d t^{\prime}$ & $F_{d 3}$ \\
$\Delta\left(\mathcal{S} \times \mathcal{S}^{\prime}\right) \times\{t=0\}, d t$ & $F_{d 20}$ \\
$\Delta\left(\mathcal{S}^{\prime} \times \mathcal{S}^{\prime \prime}\right) \times\left\{t^{\prime}=0\right\}, d t^{\prime}$ & $F_{d 02}$ \\
$\Delta\left(\mathcal{S} \times \mathcal{S}^{\prime \prime}\right) \times\left\{t^{\prime \prime}=0\right\}, d t^{\prime \prime}$ & $F_{d 22}$ \\
\hline
\end{tabular}

Let $\beta^{*} H_{2}$ be the lift of $H_{2}$ to $\mathcal{H}_{c}^{3}$, and let $\beta^{*} K_{2}$ be the lift of $K_{2}$ to $\mathcal{H}_{c}^{3}$. Then, $\beta^{*} K_{2}$ vanishes to infinite order at all boundary faces except those arising from the lift of $F_{0101}$. Now let

$$
H_{3}:=\beta_{*}\left(\beta^{*} H_{2}-\beta^{*} H_{2} \beta^{*} K_{2}\right)
$$

where $\beta_{*}$ is the push forward to $\mathcal{H}$ from $\mathcal{H}_{c}^{3}$. Since $\beta^{*} K_{2}$ vanishes to infinite order at all boundary faces except $F_{0101}$, the push forward of $\left(\beta^{*} H_{2}\right)\left(\beta^{*} K_{2}\right)$ to $\mathcal{H}$ vanishes to infinite order at all boundary faces except $F_{0101}$, where the result is given by the conic heat calculus composition rule. Consequently,

$$
H_{3}=H_{2}-\beta_{*}\left(\beta^{*} H_{2} \beta^{*} K_{2}\right)
$$

vanishes to higher order at the boundary faces of $F_{0101}$ by the conic heat calculus composition rule. Continuing this construction and using the Borel summation, we arrive at $H_{\infty}$ with expansion asymptotic to $H_{2}, H_{3}, \ldots$ and satisfying

$$
\left(\partial_{\tau}+\Delta_{0}\right) H_{\infty}=K_{\infty}
$$

where $K_{\infty}$ now vanishes to infinite order on $F_{0101}$. Using a smooth cutoff function, we now have $H_{\infty}$ defined on all of $\mathcal{H}$ satisfying

$$
\left(\partial_{t}+\Delta_{\epsilon}\right) H_{\infty}=K_{\infty}
$$

where $K_{\infty}$ vanishes to infinite order at all boundary faces of $\mathcal{H}$. 
7.3.6. Error term approximation For each $\epsilon>0$, let $E\left(z, z^{\prime}, t, \epsilon\right)=$ $H_{\epsilon}\left(z, z^{\prime}, t\right)-H_{1}\left(z, z^{\prime}, t, \epsilon\right)$. Let $K$ be defined for each $\epsilon>0$ by

$$
\left(\partial_{t}+\Delta_{\epsilon}\right) E\left(z, z^{\prime}, t, \epsilon\right)=K\left(z, z^{\prime}, t, \epsilon\right) .
$$

By construction of $H_{1}, K=O\left(\epsilon t^{\infty}\right)$ as $\epsilon, t \rightarrow 0$, so for any $N \in \mathbb{N}$, there is $C>0$ such that for any $\left(z, z^{\prime}\right) \in M \times M$,

$$
\left|K\left(z, z^{\prime}, t, \epsilon\right)\right|<C \epsilon t^{N} .
$$

Moreover, $K$ has a polyhomogeneous expansion down to $\epsilon=0$.

For each $\epsilon>0, E$ is smooth on $\mathcal{H}$ for $t>0$ by parabolic regularity applied for each $\epsilon>0$ since $K$ is $O\left(t^{\infty}\right)$. By construction, $E$ is smooth down to $t=0$, so $E\left(z, z^{\prime}, t, \epsilon\right)$ is smooth on the blown-down space, $M \times M \times \mathbb{R}^{+} \times$ $(0,1]_{\epsilon}$. The following maximum principle argument on $M \times[0, T]_{t}$ shows that $E$ is also $O\left(\epsilon t^{\infty}\right)$ as $\epsilon, t \rightarrow 0$ in the same sense as $K$.

Fix $\epsilon>0, z^{\prime} \in M$. Since $K=O\left(\epsilon t^{\infty}\right)$, fix $C>1$ and $\mathbb{N} \ni N>>1$ such that $\left|K\left(z, z^{\prime}, t, \epsilon\right)\right|^{2} \leq C \epsilon^{2} t^{2 N}$ for all $z \in M$. Let $u(z, t)=\left|E\left(z, z^{\prime}, t, \epsilon\right)\right|^{2}$. Let $\Delta$ be the scalar Laplacian for $\left(M, g_{\epsilon}\right)$. Then $u$ satisfies

$$
\begin{aligned}
\left(\partial_{t}+\Delta\right) u & =2\left\langle\left(\partial_{t}+\nabla^{*} \nabla\right) E, E\right\rangle-|\nabla E|^{2}=2\langle K-\mathcal{R} E, E\rangle-|\nabla E|^{2} \\
& \leq 2\langle K, E\rangle \leq 2|K||E| \leq|K|^{2}+|E|^{2}=|K|^{2}+u .
\end{aligned}
$$

Above we have used the positivity of $\mathcal{R}$ and the compatibility of the bundle connection with the metric. Now, let $\tilde{u}=e^{-t} u$. Then $\tilde{u}$ satisfies

$$
\left(\partial_{t}+\Delta\right) \tilde{u} \leq e^{-t}|K|^{2} \leq C \epsilon^{2} t^{2 N} .
$$

Let $w=\tilde{u}-C \epsilon^{2} t^{2 N+1}$. Since $E$ and hence $u$ and $\tilde{u}$ vanish at $t=0,\left.w\right|_{t=0}=0$, and $w$ satisfies

$$
\left(\partial_{t}+\Delta\right) w \leq C \epsilon^{2} t^{2 N}-C(2 N+1) \epsilon^{2} t^{2 N}<0 .
$$

Fix $T>0$ and consider $w$ on $M \times[0, T]_{t}$. If $w$ has a local maximum for $z \in M$ and $t \in(0, T)$, then

$$
\left(\partial_{t}+\Delta\right) w>0
$$

and this is a contradiction. If $w$ has a maximum at $t=T$, then $\partial_{t} w \geq 0$, and

$$
\left(\partial_{t}+\Delta\right) w>0
$$


which is again a contradiction. Therefore, the maximum of $w$ occurs at $t=0$ and so

$$
w \leq C \epsilon^{2} t^{2 N+1}
$$

This implies

$$
u \leq e^{T} C \epsilon^{2} t^{2 N}, \quad \text { for } 0<t \leq T,
$$

which in turn implies that $E=O\left(\epsilon t^{N}\right)$ as $\epsilon, t \rightarrow 0$, for any $N \in \mathbb{N}$.

7.3.7. Solving away the residual error term To complete this construction, we must remove the residual error term which vanishes to infinite order at the boundary faces of $\mathcal{H}$. It is now convenient to consider the elements of the acc heat calculus as $t$-convolution operators acting on $\mathcal{S} \times \mathbb{R}^{+}$. For an element $A$ which vanishes to infinite order at the boundary faces of $\mathcal{H}$ and $u$, a smooth half-density section of $\mathcal{S} \times \mathbb{R}^{+}$, the $t$-action of $A$ on $u$ is

$$
A u(t)=\int_{0}^{t}\langle A u(t-s), u(s)\rangle d s
$$

where the spatial variables have been suppressed. As a function of $s^{\prime}, s \geq 0$, $\left[A u\left(s^{\prime}\right)\right](s)$ vanishes to infinite order at $s^{\prime}=0$. Restricting to $s^{\prime}=t-s$,

$$
[A u(t-s)](s)=s^{-k / 2-1}(t-s)^{j} u_{k, j}(t-s, s)
$$

for any $-k, j \in \mathbb{N}_{0}$, where $u_{k, j}$ is a smooth half-density, so for any $-k \geq 1$ this is integrable. Consequently, $A u(t)$ as in (7.5) is smooth in $t$ and vanishes rapidly as $t \rightarrow 0$. So, an element $A$ of the acc heat calculus which vanishes to infinite order at all boundary faces of $\mathcal{H}$ gives rise to a Volterra operator. Since as a $t$-convolution operator we have

$$
\left(\partial_{t}+\Delta\right) H_{\infty}=I d-K_{\infty}
$$

we would like to invert $\left(\mathrm{Id}-K_{\infty}\right)$. Formally, the inverse should be

$$
\left(\mathrm{Id}-K_{\infty}\right)^{-1}=\sum_{j \geq 0} K_{\infty}^{j}
$$

where $K_{\infty}^{j}$ is the $j$-fold composition of $K_{\infty}$. To show that this Neumann series converges, we estimate the kernel of $K_{\infty}^{j}$. Since $K_{\infty}$ vanishes to infinite order at all boundary faces of $\mathcal{H}$, we may restrict to submanifolds of $\mathcal{H}$, estimating as in [33] and then combine these estimates to estimate $K_{\infty}^{j}$ 
on $\mathcal{H}$. The kernel $k_{\infty}^{j}$ of the restriction of $K_{\infty}^{j}$ to $M \times M \times \mathbb{R}^{+} \times\{\epsilon\}$ is bounded by

$$
\left|k_{\infty}^{j}\left(z, z^{\prime}, t, \epsilon\right)\right| \leq C_{\epsilon, j} \frac{t^{j}}{(j+1) !}, \quad t<T .
$$

This follows from the composition rule for the heat calculus on $M$ and the analogous bound in $[33,7.3]$, where we have taken $k=-2$, with $k$ as above, which we are free to choose since $A$ vanishes to infinite order. Similarly, by the composition rule for the heat calculus on $M_{0}$ and the same estimate of [33], the kernel of the restriction of $K_{\infty}^{j}$ to $M_{0} \times M_{0} \times \mathbb{R}^{+}$is bounded by

$$
\left|k_{\infty}^{j}\left(z, z^{\prime}, t\right)\right|_{F_{0101}} \leq C_{0, j} \frac{t^{j}}{(j+1) !}, \quad t<T .
$$

Similarly, the kernel of the restriction of $K_{\infty}^{j}$ to $\bar{Z} \times \bar{Z} \times \mathbb{R}_{\tau}^{+}$is bounded by

$$
\left|k_{\infty}^{j}\left(z, z^{\prime}, t\right)\right|_{F_{1010,2}} \leq C_{z, j} \frac{t^{j}}{(j+1) !}, \quad t<T .
$$

These three bounds imply that the constants $C_{\epsilon, j}$ stay bounded as $\epsilon \rightarrow 0$, and so we have the following global bound for the kernel of $K_{\infty}^{j}$ on both $\mathcal{H}$ and the blown-down space $\left\{\epsilon=\epsilon^{\prime}\right\} \subset \mathcal{S} \times \mathcal{S} \times \mathbb{R}^{+}$

$$
\left|k_{\infty}^{j}\left(z, z^{\prime}, t, \epsilon\right)\right| \leq C_{j} \frac{t^{j}}{(j+1) !}, \quad t<T .
$$

It follows that the Neumann series for $\left(\mathrm{Id}-K_{\infty}\right)^{-1}$ is summable and has an inverse which as a $t$-convolution operator is also of the form $(\operatorname{Id}-A)$, where $A$ is an element of the acc heat calculus that vanishes to infinite order at the boundary faces of $\mathcal{H}$. Then, the full acc heat kernel is

$$
H=H_{\infty}\left(\mathrm{Id}-K_{\infty}\right)^{-1}
$$

As a consequence of this construction, $H$ has a fully polyhomogeneous expansion down to $\epsilon=0$ with leading order terms given by the acc model heat kernel, $H_{1}$. 
Remarks. A consequence of this theorem is the uniform convergence for $0<t<T$,

$$
H_{\epsilon} \rightarrow H_{0}+O(\epsilon) \text { as } \epsilon \rightarrow 0 \text {. }
$$

This extends the convergence results of [11] for scalar heat kernels to heat kernels for geometric Laplacians acting on vector bundles. Due to the rescaled time variables at the faces contained in $\epsilon, t=0$, we expect to see interesting applications involving the short time asymptotic behavior of the heat trace; see [31]. We have not computed the $t \rightarrow \infty$ asymptotics but expect this will also yield interesting applications.

\section{A. Asymptotically conic scattering heat kernel}

Let $\left(\bar{Z}, g_{z}\right)$ be an ac scattering metric with boundary defined by $\{x=0\}$ and local coordinates $(x, y)$ near the boundary. Let $\Delta_{z}$ be the Friedrichs extension of a geometric Laplacian on $\bar{Z}$. We motivate the definition of the acc heat space by lifting the Euclidean heat kernel to $\bar{Z}_{+}^{2}$.

Recall the Euclidean heat kernel for $\mathbb{R}^{n}$,

$$
G\left(z, z^{\prime}, t\right)=(4 \pi t)^{-n / 2} e^{-\left|z-z^{\prime}\right|^{2} / 2 t}
$$

Consider the coordinates $z=(r, y)$ on the complete ac space. With the compactification given by $x=\frac{1}{r}$ in the local coordinates $\left(x, y, x^{\prime}, y^{\prime}, t\right)$ on $\bar{Z}_{+}^{2}$ near the boundary of $\bar{Z}$, the Euclidean heat kernel is

$$
G\left(x, y, x^{\prime}, y^{\prime}, t\right)=(4 \pi t)^{-n / 2} \exp \left(-\frac{\left(\left|(1 / x)-\left(1 / x^{\prime}\right)\right|^{2}+\left|y-y^{\prime}\right|^{2}\right)}{2 t}\right) .
$$

This motivates blowing up

$$
\left\{\left(x, y, x^{\prime}, y^{\prime}, t\right): x=0, x^{\prime}=0\right\}
$$

In the projective coordinates $s=\frac{x}{x^{\prime}}$ and $s^{\prime}=x^{\prime}$, the Euclidean heat kernel is

$$
G\left(s, y, s^{\prime}, y^{\prime}, t\right)=(4 \pi t)^{-n / 2} \exp \left(-\frac{\left(\left|(s-1) / s s^{\prime}\right|^{2}+\left|y-y^{\prime}\right|^{2}\right)}{2 t}\right) .
$$

This motivates a second blowup at $s=1$, along the submanifold where the diagonal in $\bar{Z} \times \bar{Z}$ meets the first blown-up face

$$
\left\{\left(x, y, x^{\prime}, y^{\prime}, t\right): x=0, x^{\prime}=0, y=y^{\prime}\right\}
$$




\section{A.1. Asymptotically conic scattering heat space}

As motivated above, the ac scattering heat space is constructed from $\bar{Z}_{+}^{2}$ by performing three blowups.

The scattering double space, $\bar{Z}_{\mathrm{sc}}^{2}$, originally constructed by [17], is

$$
\bar{Z}_{\mathrm{sc}}^{2}:=\left[[\bar{Z} \times \bar{Z} ; \partial \bar{Z} \times \partial \bar{Z}] ; \Delta(Y \times Y) \cap F_{110}\right]
$$

where $F_{110}$ is the face created by the first blowup. Including the time variable, we perform one more blowup to construct the ac scattering heat space,

$$
\bar{Z}_{\mathrm{sc}, h}^{2}=\left[\bar{Z}_{\mathrm{sc}}^{2} \times \mathbb{R}^{+} ; \Delta(Z \times Z) \times\{t=0\}, d t\right]
$$

The ac scattering heat space has six boundary faces described in the following table.

\begin{tabular}{lll}
\hline Face & Geometry of face & Defining function in local coordinates \\
\hline$F_{220}$ & $N^{+}(\Delta(Y \times Y)) \times \mathbb{R}^{+}$ & $\rho_{220}=\left(x^{2}+\left(x^{\prime}\right)^{2}+\left|y-y^{\prime}\right|^{2}\right)^{1 / 2}$ \\
$F_{220}$ & $N^{+}((Y \times Y)$ & \\
& $-\Delta(Y \times Y)) \times \mathbb{R}^{+}$ & $\rho_{110}=\left(x^{2}+\left(x^{\prime}\right)^{2}\right)^{1 / 2}$ \\
$F_{100}$ & $Z \times Y \times \mathbb{R}^{+}$ & $\rho_{100}=x$ \\
$F_{010}$ & $Y \times Z \times \mathbb{R}^{+}$ & $\rho_{010}=x^{\prime}$ \\
$F_{d 2}$ & $P N_{t}^{+}(\Delta(Z \times Z))$ & $\rho_{d 2}=\left(\left|z-z^{\prime}\right|^{4}+t^{2}\right)^{1 / 2}$ \\
$F_{001}$ & $(Z \times Z)-\Delta(Z \times Z)$ & $\rho_{001}=t$ \\
\hline
\end{tabular}

\section{A.2. Asymptotically conic scattering heat calculus}

Elements of the ac scattering heat calculus are distributional section halfdensities on $\bar{Z}_{+}^{2}$ which are smooth on the interior and lift to be polyhomogeneous on $\bar{Z}_{\mathrm{sc}, h}^{2}$. Let $\mu$ be a smooth, non-vanishing half-density on $\bar{Z} \times \bar{Z} \times \mathbb{R}^{+}$, and let $\nu$ be a smooth, non-vanishing half-density on $\bar{Z}_{\mathrm{sc}, h}^{2}$.

Definition A.1. For any $k \in \mathbb{R}$ and index sets $E_{110}$ and $E_{220}, A \in$ $\Psi_{\mathrm{sc}, H}^{E_{110}, E_{220}, k}$, if $A$ is smooth on the interior of $\bar{Z}_{\mathrm{sc}, h}^{2}$ and satisfies the following.

1. $A \in \mathcal{A}_{p h g}^{-(1 / 2)+E_{110}}$ at $F_{110}$.

2. $A \in \mathcal{A}_{p h g}^{(-(n+2) / 2)+E_{220}}$ at $F_{220}$. 
3. $A$ vanishes to infinite order at $F_{001}, F_{100}$ and $F_{010}$.

4. $A \in \rho_{d 2}^{-((n+3) / 2)-k} \mathcal{C}^{\infty}\left(F_{d 2}\right)$.

Elements of the ac scattering heat calculus act on sections of $\bar{Z}$ in the usual way and on sections of $Z \times \mathbb{R}_{t}^{+}$by $t$-convolution. The composition rule is proven using the ac scattering triple heat space, $\bar{Z}_{\mathrm{sc}, h}^{3}$. This space has partial blow-down and projection maps to three identical copies of the ac scattering heat space as well as full blow-down and projection maps to three identical copies of $\bar{Z}_{+}^{2}$; these are called the left, right and center. Formally, two elements of the ac heat calculus are composed by lifting from the left and right copies of $\bar{Z}_{\mathrm{sc}, h}^{2}$ to $\bar{Z}_{\mathrm{sc}, h}^{3}$, then multiplying and blowing down/projecting to the center copy of $\bar{Z}_{\mathrm{sc}, h}^{2}$. It is key that the triple space be constructed so that these lifts and push-forward maps are $b$-fibrations in order that polyhomogeneity be preserved.

A.2.1. Asymptotically conic scattering triple heat space In a neighborhood of the boundary in each copy of $\bar{Z}$, we have the local coordinates $(x, y)$, which provide the local coordinates $\left(x, y, x^{\prime}, y^{\prime}, x^{\prime \prime}, y^{\prime \prime}\right)$ on $\bar{Z}^{3}$. First, we blow up the codimension 3 corner defined by $\left\{x=0, x^{\prime}=0, x^{\prime \prime}=0\right\}$, resulting in the face $F_{11100}$ with defining function locally given by

$$
\rho_{11100}=\left(x^{2}+\left(x^{\prime}\right)^{2}+\left(x^{\prime \prime}\right)^{2}\right)^{1 / 2} .
$$

Next, we blow up the three codimension 2 corners corresponding to the $F_{110}$ faces in each of the three copies of $\bar{Z}_{\mathrm{sc}, h}^{2}$. These faces are as follows.

\begin{tabular}{lll}
\hline Face & Submanifold to be blown up & Defining function \\
\hline$F_{11000}$ & $S_{11000}=\left\{x=0, x^{\prime}=0\right\}-F_{11100}$ & $\rho_{11000}=\left(x^{2}+\left(x^{\prime}\right)^{2}\right)^{1 / 2}$ \\
$F_{01100}$ & $S_{01100}=\left\{x^{\prime}=0, x^{\prime \prime}=0\right\}-F_{11100}$ & $\rho_{01100}=\left(\left(x^{\prime}\right)^{2}+\left(x^{\prime \prime}\right)^{2}\right)^{1 / 2}$ \\
$F_{10100}$ & $S_{10100}=\left\{x=0, x^{\prime \prime}=0\right\}-F_{11100}$ & $\rho_{10100}=\left((x)^{2}+\left(x^{\prime \prime}\right)^{2}\right)^{1 / 2}$ \\
\hline
\end{tabular}

Next we blow up the codimension $2 n+1$ corner where the diagonals meet $F_{11100}$. After the $F_{11100}$ blowup, we have coordinates $\left(\theta, \theta^{\prime}, \theta^{\prime \prime}, y, y^{\prime}, y^{\prime \prime}\right.$, $\left.\rho_{11100}\right)$, with

$$
x=\left(\rho_{11100}\right) \theta, \quad x^{\prime}=\left(\rho_{11100}\right) \theta^{\prime}, \quad x^{\prime \prime}=\left(\rho_{11100}\right) \theta^{\prime \prime}, \quad(\theta)^{2}+\left(\theta^{\prime}\right)^{2}+\left(\theta^{\prime \prime}\right)^{2}=1 .
$$


Using these coordinates, we next blow up

$$
S_{22200}=\left\{\theta=\theta^{\prime}=\theta^{\prime \prime}, y=y^{\prime}=y^{\prime \prime}, r_{0}=0\right\}
$$

The face created by this blowup is called $F_{22200}$ with defining function

$$
\rho_{22200}=\left(\left(\theta-\theta^{\prime}\right)^{2}+\left(\theta^{\prime}-\theta^{\prime \prime}\right)^{2}+\left|y-y^{\prime}\right|^{2}+\left|y^{\prime}-y^{\prime \prime}\right|^{2}+r_{0}^{2}\right)^{1 / 2} .
$$

After this, we blow up the three codimension $n$ corners corresponding to the $F_{220}$ faces in the three copies of the double heat space. These are as

\begin{tabular}{|c|c|c|}
\hline Face & Submanifold to be blown up & Defining function \\
\hline$\overline{F_{22000}}$ & $S_{22000}=\left\{\theta=0, \theta^{\prime}=0, y=y^{\prime}\right\}$ & $\begin{aligned} \rho_{22000}= & \left(\theta^{2}+\left(\theta^{\prime}\right)^{2}\right. \\
& \left.+\left|y-y^{\prime}\right|^{2}\right)^{1 / 2}\end{aligned}$ \\
\hline$F_{02200}$ & $S_{02200}=\left\{\theta^{\prime}=0, \theta^{\prime \prime}=0, y^{\prime}=y^{\prime \prime}\right\}$ & $\begin{aligned} \rho_{02200}= & \left(\left(\theta^{\prime}\right)^{2}+\left(\theta^{\prime \prime}\right)^{2}\right. \\
& \left.+\left|y^{\prime}-y^{\prime \prime}\right|^{2}\right)^{1 / 2}\end{aligned}$ \\
\hline$F_{20200}$ & $S_{20200}=\left\{\theta=0, \theta^{\prime \prime}=0, y=y^{\prime \prime}\right\}$ & $\begin{aligned} \rho_{20200}= & \left((\theta)^{2}+\left(\theta^{\prime \prime}\right)^{2}\right. \\
& \left.+\left|y-y^{\prime \prime}\right|^{2}\right)^{1 / 2}\end{aligned}$ \\
\hline
\end{tabular}
follows.

We have now constructed the ac scattering triple space, $\bar{Z}_{\mathrm{sc}}^{3}$. We next introduce the time variables and perform the parabolic temporal diagonal blowups. We must first blow up the codimension 2 corner of $\mathbb{R}^{+} \times \mathbb{R}^{+}$to preserve symmetry. Let

$$
\mathcal{T}_{0}^{2}=\left[\mathbb{R}^{+} \times \mathbb{R}^{+} ; t=t^{\prime}=0\right]
$$

The defining function for the blowup of $\left\{t=t^{\prime}=0\right\}$ is $\rho_{00011}$, which we call $t^{\prime \prime}$ because it plays the role of the third time variable. We now take $Z_{\mathrm{sc}}^{3} \times \mathcal{T}_{0}^{2}$ and blow up the temporal diagonal faces. First, we blow up the codimension $2 n+3$ triple diagonal, $S_{d 3}$, defined by

$$
\left\{z=z^{\prime}=z^{\prime \prime}, t^{\prime \prime}=0\right\} .
$$

The defining function of this face is $\rho_{d 3}$,

$$
\rho_{d 3}=\left(\left|z-z^{\prime}\right|^{4}+\left|z-z^{\prime \prime}\right|^{4}+\left(t^{\prime \prime}\right)^{2}\right)^{1 / 4} .
$$


Next, we blow up the three temporal diagonals corresponding to the diagonal faces in the three copies of the double heat space. These are as follows.

\begin{tabular}{lll}
\hline Face & Submanifold to be blown up & Defining function \\
\hline$F_{d 20}$ & $S_{d 20}=\left\{z=z^{\prime}\right\}$ & $\rho_{d 20}=\left(\left|z-z^{\prime}\right|^{4}+t^{2}\right)^{1 / 4}$ \\
$F_{d 02}$ & $S_{d 02}=\left\{z^{\prime}=z^{\prime \prime}\right\}$ & $\rho_{d 02}=\left(\left|z^{\prime}-z^{\prime \prime}\right|^{4}+\left(t^{\prime}\right)^{2}\right)^{1 / 4}$ \\
$F_{d 22}$ & $S_{d 22}=\left\{z=z^{\prime \prime}\right\}$ & $\rho_{d 22}=\left(\left|z-z^{\prime \prime}\right|^{4}+\left(t^{\prime \prime}\right)^{2}\right)^{1 / 4}$ \\
\hline
\end{tabular}

We have now constructed the ac scattering triple heat space and proceed with the composition rule.

Technical Theorem A.2. Let $A \in \Psi_{\mathrm{sc}, H}^{A_{110}, A_{220}, k_{a}}$ and $B \in \Psi_{\mathrm{sc}, H}^{B_{110}, B_{220}, k_{b}}$. Then, the composition $B \circ A$ is an element of $\Psi_{\mathrm{sc}, H}^{A_{110} \bar{\cup} B_{110}, A_{220} \bar{\cup} B_{220}, k_{a}+k_{b}}$.

A.2.2. Proof. Formally we have,

$$
\kappa_{B \circ A} \nu=\left(\beta_{\mathrm{C}}\right)_{*}\left(\left(\beta_{\mathrm{R}}\right)^{*}\left(\kappa_{A} \nu\right)\left(\beta_{\mathrm{L}}\right)^{*}\left(\kappa_{B} \nu\right)\right)
$$

Multiplying both sides of (A.1) by $\nu$ and using the fact that $\left(\beta_{c}\right)_{*}\left(\beta_{c}\right)^{*}$ $(\nu)=\nu$,

$$
\kappa_{B \circ A} \nu^{2}=\left(\beta_{\mathrm{C}}\right)_{*}\left(\left(\beta_{\mathrm{R}}\right)^{*}\left(\kappa_{A} \nu\right)\left(\beta_{\mathrm{L}}\right)^{*}\left(\kappa_{B} \nu\right)\left(\beta_{c}\right)^{*}(\nu)\right) .
$$

Next we calculate the lifts of the defining functions and half densities from $\bar{Z}_{\mathrm{sc}, h}^{2}$ to $\bar{Z}_{\mathrm{sc}, h}^{3}$. A calculation gives the half density on the heat space $\nu$ in terms of the half density $\mu$ on $\bar{Z}_{+}^{2}$,

$$
\nu=\left(\beta_{h}\right)^{*}\left(\left(\rho_{110}\right)^{-1 / 2}\left(\rho_{220}\right)^{-n / 2}\left(\rho_{d 2}\right)^{-n+1 / 2} \mu\right) .
$$

The ac scattering triple heat space has partial blow-down/projection maps $\beta_{\mathrm{L}}, \beta_{\mathrm{R}}$ and $\beta_{\mathrm{C}}$ to three identical copies of $\bar{Z}_{\mathrm{sc}, h}^{2}$. If we denote the three copies of $\bar{Z}$ by $\bar{Z}, \bar{Z}^{\prime}, \bar{Z}^{\prime \prime}$ and the three time variables $\left(t, t^{\prime}, t^{\prime \prime}\right)$, where $t^{\prime \prime}$ is from the blowup of $\mathbb{R}^{+} \times \mathbb{R}^{+}$, then the three copies of $\bar{Z}_{\mathrm{sc}, h}^{2}$ are as follows.

\begin{tabular}{ll}
\hline Copy of $\bar{Z}_{\mathrm{sc}, h}^{2}$ & Associated to in $\bar{Z}_{\mathrm{sc}, h}^{3}$ \\
\hline Left & $\bar{Z} \times \bar{Z}^{\prime} \times \mathbb{R}_{t}^{+}$ \\
Right & $\bar{Z}^{\prime} \times \bar{Z}^{\prime \prime} \times \mathbb{R}_{t^{\prime}}^{+}$ \\
Center & $\bar{Z} \times \bar{Z}^{\prime \prime} \times \mathbb{R}_{t^{\prime \prime}}^{+}$ \\
\hline
\end{tabular}


Next, we compute the lifts of the defining functions for the boundary faces of the heat space to the triple heat space.

\begin{tabular}{lll}
\hline Lifting map & Defining function on $\bar{Z}_{\mathrm{sc}, h}^{2}$ & Lift to $\bar{Z}_{\mathrm{sc}, h}^{3}$ \\
\hline$\left(\beta_{\mathrm{L}}\right)^{*}$ & $\rho_{100}$ & $\rho_{10000} \rho_{10100}$ \\
$\left(\beta_{\mathrm{L}}\right)^{*}$ & $\rho_{010}$ & $\rho_{01000} \rho_{01100}$ \\
$\left(\beta_{\mathrm{L}}\right)^{*}$ & $\rho_{110}$ & $\rho_{11100} \rho_{11000}$ \\
$\left(\beta_{\mathrm{L}}\right)^{*}$ & $\rho_{220}$ & $\rho_{22200} \rho_{22000}$ \\
$\left(\beta_{\mathrm{L}}\right)^{*}$ & $\rho_{d 2}$ & $\rho_{d 3} \rho_{d 20}$ \\
$\left(\beta_{\mathrm{L}}\right)^{*}$ & $\rho_{001}$ & $\rho_{00010} \rho_{00011} \rho_{d 22}$ \\
$\left(\beta_{\mathrm{R}}\right)^{*}$ & $\rho_{100}$ & $\rho_{01000} \rho_{01100}$ \\
$\left(\beta_{\mathrm{R}}\right)^{*}$ & $\rho_{010}$ & $\rho_{00100} \rho_{10100}$ \\
$\left(\beta_{\mathrm{R}}\right)^{*}$ & $\rho_{110}$ & $\rho_{11100} \rho_{01100}$ \\
$\left(\beta_{\mathrm{R}}\right)^{*}$ & $\rho_{220}$ & $\rho_{22200} \rho_{02200}$ \\
$\left(\beta_{\mathrm{R}}\right)^{*}$ & $\rho_{d 2}$ & $\rho_{d 3} \rho_{d 02}$ \\
$\left(\beta_{\mathrm{R}}\right)^{*}$ & $\rho_{001}$ & $\rho_{00001} \rho_{00011} \rho_{d 22}$ \\
$\left(\beta_{\mathrm{C}}\right)^{*}$ & $\rho_{100}$ & $\rho_{10000} \rho_{11000}$ \\
$\left(\beta_{\mathrm{C}}\right)^{*}$ & $\rho_{010}$ & $\rho_{001000} \rho_{01100}$ \\
$\left(\beta_{\mathrm{C}}\right)^{*}$ & $\rho_{110}$ & $\rho_{11100} \rho_{10100}$ \\
$\left(\beta_{\mathrm{C}}\right)^{*}$ & $\rho_{220}$ & $\rho_{22200} \rho_{20200}$ \\
$\left(\beta_{\mathrm{C}}\right)^{*}$ & $\rho_{d 2}$ & $\rho_{d 3} \rho_{d 22}$ \\
$\left(\beta_{\mathrm{C}}\right)^{*}$ & $\rho_{001}$ & $\rho_{00022} \rho_{00011} \rho_{d 22}$ \\
\hline & &
\end{tabular}

Then,

$$
\left(\beta_{\mathrm{L}}\right)^{*}(\nu)=\left(\beta_{\mathrm{L}}\right)^{*}\left(\left(\rho_{110}\right)^{-1 / 2}\left(\rho_{220}\right)^{-n / 2}\left(\rho_{d 2}\right)^{-n+1 / 2} \mu\right) .
$$

Next, we use the fact that

$$
\left(\beta_{\mathrm{L}}\right)^{*}(\mu)\left(\beta_{\mathrm{R}}\right)^{*}(\mu)\left(\beta_{\mathrm{C}}\right)^{*}(\mu)=\mu_{3}^{2}
$$

Here, $\mu_{3}^{2}$ is a smooth density on $\bar{Z} \times \bar{Z} \times \bar{Z} \times \mathbb{R}^{+} \times \mathbb{R}^{+}$, so we may assume

$$
\mu_{3}^{2}=d z d z^{\prime} d z^{\prime \prime} d t d t^{\prime}
$$

A Jacobian calculation gives the lift of $\mu_{3}^{2}$ to the triple heat space. First note

$$
\left(\beta_{3}\right)^{*}(x)=\left(\rho_{11100}\right)\left(\rho_{11000}\right)\left(\rho_{10100}\right)\left(\rho_{10000}\right),
$$




$$
\begin{aligned}
\left(\beta_{3}\right)^{*}\left(x^{\prime}\right) & =\left(\rho_{11100}\right)\left(\rho_{11000}\right)\left(\rho_{01100}\right)\left(\rho_{01000}\right), \\
\left(\beta_{3}\right)^{*}\left(x^{\prime \prime}\right) & =\left(\rho_{11100}\right)\left(\rho_{01100}\right)\left(\rho_{10100}\right)\left(\rho_{00100}\right) .
\end{aligned}
$$

This implies

$$
\begin{gathered}
\left(\beta_{3}\right)^{*}\left(\mu_{3}^{2}\right)=\left(\rho_{11100}\right)^{2}\left(\rho_{11000} \rho_{01100} \rho_{10100}\right)\left(\rho_{22000} \rho_{02200} \rho_{20200}\right)^{n} \\
\left(\rho_{22200}\right)^{2 n+1}\left(\rho_{d 20} \rho_{d 02} \rho_{d 22}\right)^{n+1} \rho_{d 3}^{2 n+3}\left(t^{\prime \prime}\right) \nu_{3}^{2} .
\end{gathered}
$$

Here, $\nu_{3}^{2}$ is a smooth, non-vanishing density on the triple heat space. Combining this with the above lifts, we arrive at the following formula

$$
\begin{gathered}
\left(\beta_{\mathrm{L}}\right)^{*}(\nu)\left(\beta_{\mathrm{R}}\right)^{*}(\nu)\left(\beta_{\mathrm{C}}\right)^{*}(\nu)=\left(\rho_{11100}\right)^{1 / 2}\left(\rho_{10100} \rho_{01100} \rho_{10100}\right)^{1 / 2} \\
\left(\rho_{22000} \rho_{02200} \rho_{20200}\right)^{n / 2}\left(\rho_{22200}\right)^{(n+1) / 2}\left(\rho_{d 3}\right)^{(n+3) / 2}\left(\rho_{d 20} \rho_{d 02} \rho_{d 22}\right)^{(n+1) / 2}\left(t^{\prime \prime}\right) \nu_{3}^{2} .
\end{gathered}
$$

To use the push-forward theorem of [28], we need to write each of these in terms of $b$-densities. First, we have on the center copy of $\bar{Z}_{\mathrm{sc}, h}^{2}$,

$$
{ }^{b} \nu^{2}=\left(\rho_{100} \rho_{010} \rho_{110} \rho_{220} \rho_{001} \rho_{d 2}\right)^{-1} \nu^{2} .
$$

Then, we have

$$
{ }^{b} \nu^{2}=\left(\beta_{c}\right)_{*}\left(\beta_{c}\right)^{*}\left(\left(\rho_{100} \rho_{010} \rho_{110} \rho_{220} \rho_{001} \rho_{d 2}\right)^{-1} \nu^{2}\right) .
$$

We observe

$$
\left(\beta_{c}\right)^{*}\left(\left(\rho_{100} \rho_{010} \rho_{110} \rho_{220} \rho_{001} \rho_{d 2}\right)^{-1}\right)=
$$

$\left(\rho_{10000} \rho_{00100} \rho_{11000} \rho_{01100} \rho_{10100} \rho_{11100} \rho_{22200} \rho_{02200} \rho_{20200} \rho_{d 3} \rho_{d 22} \rho_{00011}\right)^{-1}$.

So now we multiply both sides of $(\mathrm{A} .2)$ by $\left(\beta_{c}\right)_{*}\left(\beta_{c}\right)^{*}\left(\rho_{100} \rho_{010} \rho_{110} \rho_{220}\right.$ $\left.\rho_{001} \rho_{d 2}\right)^{-1}$ ) and inside the right side of (A.2) we have

$$
\begin{gathered}
\left(\rho_{11100} \rho_{11000} \rho_{01100} \rho_{10100}\right)^{-1 / 2}\left(\rho_{22000} \rho_{02200} \rho_{22200}\right)^{n / 2}\left(\rho_{20200}\right)^{(n-2) / 2} \\
\left(\rho_{d 3}\right)^{(n+1) / 2}\left(\rho_{d 20} \rho_{d 02}\right)^{(n+1) / 2}\left(\rho_{d 22}\right)^{n / 2}\left(\rho_{10000} \rho_{00100}\right)^{-1} \nu_{3}^{2} .
\end{gathered}
$$

To use the push-forward theorem, we must change the density $\nu_{3}^{2}$ to a b-density. We observe

$$
\begin{gathered}
{ }^{b} \nu_{3}^{2}=\left(\rho_{11100} \rho_{11000} \rho_{01100} \rho_{10100} \rho_{22200} \rho_{22000} \rho_{02200} \rho_{20200}\right. \\
\left.\rho_{10000} \rho_{01000} \rho_{00100} \rho_{d 3} \rho_{d 20} \rho_{d 02} \rho_{d 22} \rho_{00011} \rho_{00010} \rho_{00001}\right)^{-1} \nu_{3}^{2} .
\end{gathered}
$$


So, we now have for the composition formula

$$
\begin{gathered}
\left(\beta_{c}\right)_{*}\left(\tilde{\kappa_{A}} \tilde{\kappa_{B}}\left(\rho_{11100} \rho_{11000} \rho_{01100} \rho_{10100}\right)^{1 / 2}\left(\rho_{22200} \rho_{22000} \rho_{02200}\right)^{(n+2) / 2}\right. \\
\left.\left(\rho_{20200}\right)^{n / 2}\left(\rho_{d 3} \rho_{d 20} \rho_{d 02}\right)^{(n+3) / 2}\left(\rho_{d 22}\right)^{(n+1) / 2} \rho_{01000} \rho_{00011} \rho_{00010} \rho_{00001}\left({ }^{b} \nu_{3}^{2}\right)\right) .
\end{gathered}
$$

We observe the following orders of $\tilde{\kappa_{A}}$ on $\bar{Z}_{\mathrm{sc}, h}^{3}$.

\begin{tabular}{ll}
\hline Face & $\tilde{\kappa_{A}}$ Index set/leading order \\
\hline$F_{11100}$ & $-1 / 2+A_{110}$ \\
$F_{11000}$ & $-1 / 2+A_{220}$ \\
$F_{01100}, F_{10100}, F_{02200}, F_{20200}, F_{d 22}$ & $\infty$ \\
$F_{22200}, F_{22000}$ & $-(n+2) / 2+A_{220}$ \\
$F_{d 3}, F_{d 20}$ & $-(n+3) / 2-k_{a}$ \\
$F_{10000}, F_{01000}, F_{00100}, F_{00010}, F_{00011}$ & $\infty$ \\
\hline
\end{tabular}

Similarly, for $\tilde{\kappa_{B}}$, we have orders as follows.

\begin{tabular}{ll}
\hline Face & $\tilde{\kappa_{B}}$ Index set/leading order \\
\hline$F_{11100}$ & $-1 / 2+B_{110}$ \\
$F_{01100}$ & $-1 / 2+B_{220}$ \\
$F_{11000}, F_{10100}, F_{22000}, F_{20200}, F_{d 22}$ & $\infty$ \\
$F_{22200}, F_{02200}$ & $-(n+2) / 2+B_{220}$ \\
$F_{d 3}, F_{d 02}$ & $-(n+3) / 2-k_{b}$ \\
$F_{10000}, F_{01000}, F_{00100}, F_{00010}, F_{00011}$ & $\infty$ \\
\hline
\end{tabular}

Now, recalling the formula:

$$
\left(\beta_{c}\right)_{*}\left(\tilde{\kappa_{A}} \tilde{\kappa_{B}}\left(\rho_{11100} \rho_{11000} \rho_{01100} \rho_{10100}\right)^{1 / 2}\left(\rho_{22200} \rho_{22000} \rho_{02200}\right)^{(n+2) / 2}\right.
$$

$\left.\left(\rho_{20200}\right)^{n / 2}\left(\rho_{d 3} \rho_{d 20} \rho_{d 02}\right)^{(n+3) / 2}\left(\rho_{d 22}\right)^{(n+1) / 2} \rho_{01000} \rho_{00011} \rho_{00010} \rho_{00001}\left({ }^{b} \nu_{3}^{2}\right)\right)$.

We see that the quantity on the right-hand side to be pushed forward by $\left(\beta_{c}\right)_{*}$ has the following indices on the boundary faces.

\begin{tabular}{ll}
\hline Face & Index set/leading order \\
\hline$F_{11100}$ & $-1 / 2+A_{110} \bar{\cup} B_{110}$ \\
$F_{11000}, F_{01100}, F_{10100}, F_{22000}, F_{02200}, F_{20200}$ & $\infty$ \\
$F_{22200}$ & $-(n+2) / 2+A_{220} \cup B_{220}$ \\
$F_{d 3}$ & $-(n+3) / 2-\left(k_{a}+k_{b}\right)$ \\
$F_{d 20}, F_{d 02}, F_{d 22}$ & $\infty$ \\
$F_{10000}, F_{01000}, F_{00100}, F_{00010}, F_{00001}, F_{00011}$ & $\infty$ \\
\hline
\end{tabular}


The push forward under $\left(\beta_{c}\right)^{*}$ sends the boundary faces of $\bar{Z}_{\mathrm{sc}, h}^{3}$ to $\bar{Z}_{\mathrm{sc}, h}^{2}$ as follows.

\begin{tabular}{ll}
\hline $\bar{Z}_{h}^{3}$ face & Boundary face of $\bar{Z}_{\mathrm{sc}, h}^{2}$ or interior \\
\hline$F_{11100}$ & $F_{110}$ \\
$F_{10100}$ & $F_{110}$ \\
$F_{22200}, F_{20200}$ & $F_{220}$ \\
$F_{d 3}, F_{d 22}$ & $F_{d 2}$ \\
$F_{10000}$ & $F_{100}$ \\
$F_{00100}$ & $F_{010}$ \\
$F_{00011}$ & $F_{001}$ \\
$F_{11000}, F_{01100} F_{22000}, F_{02200}$, & Interior \\
$F_{d 20}, F_{d 02}, F_{01000}, F_{00010}, F_{00001}$ & Interior \\
\hline
\end{tabular}

The quantity to be pushed forward is integrable with respect to ${ }^{b} \nu_{3}^{2}$ at the faces that are mapped to the interior, so we may apply the pushforward theorem [28] to arrive at the result of the composition rule. The kernel, $\kappa_{B \circ A}$, will have the following polyhomogeneous index sets and leading orders on $\bar{Z}_{h}^{2}$.

\begin{tabular}{ll}
\hline Face of $\bar{Z}_{h}^{2}$ & Index set/leading order \\
\hline$F_{110}$ & $-1 / 2+A_{110} \cup \bar{\cup} B_{110}$ \\
$F_{220}$ & $-(n+2) / 2+A_{220} \cup B_{220}$ \\
$F_{d 2}$ & $-(n+3) / 2-\left(k_{a}+k_{b}\right)$ \\
$F_{100}$ & $\infty$ \\
$F_{010}$ & $\infty$ \\
$F_{001}$ & $\infty$ \\
\hline
\end{tabular}

This concludes the proof of the composition rule.

Technical Theorem A.3. Let $\left(Z, g_{z}\right)$ be an ac scattering space with bound$\operatorname{ary}(Y, h)$. Let $(E, \nabla)$ be a Hermitian vector bundle over $\left(Z, g_{z}\right)$ so that near the boundary $E$ is the pullback of a bundle over $(Y, h)$. Let $\Delta$ be a geometric Laplacian on $\left(Z, g_{z}\right)$ associated to the bundle $(E, \nabla)$. Then there exists $H \in \Psi_{\mathrm{ac}, H}^{E_{110}, E_{220},-2}$ satisfying

$$
\begin{gathered}
\left(\partial_{t}+\Delta\right) H\left(z, z^{\prime}, t\right)=0, \quad t>0 \\
H\left(z, z^{\prime}, 0\right)=\delta\left(z-z^{\prime}\right) .
\end{gathered}
$$

Moreover, $H$ vanishes to infinite order at $F_{110}$ and is smooth up to $F_{220}$. 
On the interior of $\bar{Z}_{\mathrm{ac}, h}^{2}$, the ac scattering model heat kernel is locally defined by the Euclidean heat kernel and a partition of unity. At $F_{d 2}$, we construct the model heat kernel explicitly using the jet of the metric at the base point of each fiber. At $F_{001}$, the model heat kernel vanishes to infinite order. At $F_{110}$ and $F_{220}$, the model heat kernel is the lift of the Euclidean heat kernel. Then the ac scattering model heat kernel $H_{1}$ satisfies

$$
\left(\partial_{t}+\Delta\right) H_{1}=K_{1}
$$

where $K_{1}$ vanishes to positive order on the boundary faces of $\bar{Z}_{\mathrm{sc}, h}^{2}$. We now define

$$
H_{2}=H_{1}-H_{1} K_{1}
$$

with

$$
\left(\partial_{t}+\Delta\right) H_{2}=K_{2}
$$

where $K_{2}$ vanishes to one order higher on the boundary faces of $\bar{Z}_{\mathrm{sc}, h}^{2}$. Similarly,

$$
H_{3}:=H_{2}-H_{2} K_{2}
$$

Using the Borel summation, we construct $H_{\infty}$ with expansion asymptotic to $H_{1}, H_{2}, H_{3}, \ldots$ and satisfying

$$
\left(\partial_{t}+\Delta\right) H_{\infty}=K
$$

where now $K$ vanishes to infinite order on the boundary faces of $\bar{Z}_{\mathrm{sc}, h}^{2}$. As a $t$-convolution operator, we wish to have

$$
H_{\infty}=\mathrm{Id}
$$

However, we currently have

$$
H_{\infty}=\mathrm{Id}+K
$$

but this is not a problem since $(\mathrm{Id}+K)$ is invertible with inverse of the same form. Then the ac scattering heat kernel

$$
H=H_{\infty}(\mathrm{Id}-K)^{-1}
$$

is an element of the ac scattering heat calculus with leading orders on the boundary faces of $\bar{Z}_{\mathrm{sc}, h}^{2}$ determined by those of the model kernel. 


\section{B. Asymptotically conic convergence triple heat space}

Let

$$
T:=\left[\left[\left[\left[\mathcal{S} \times \mathcal{S} \times \mathcal{S} ; Y \times Y^{\prime} \times Y^{\prime \prime}\right] ; Y \times Y^{\prime}\right] ; Y^{\prime} \times Y^{\prime \prime}\right] ; Y \times Y^{\prime \prime}\right],
$$

where we have used $Y, Y^{\prime}, Y^{\prime \prime}$ to denote the three copies of $Y$ in $\mathcal{S}^{3}$. Let

$$
\begin{aligned}
\mathcal{T}:=\left\{p \in T: f_{i}(p)=0, i=1,2\right\}, \quad f_{1}(p)=x(p) r(p)-x^{\prime}(p) r^{\prime}(p), \\
\\
f_{2}(p)=x^{\prime}(p) r^{\prime}(p)-x^{\prime \prime}(p) r^{\prime \prime}(p) .
\end{aligned}
$$

Like the acc double and heat space, $\mathcal{T}$ is a smooth manifold with corners. Let

$$
\mathbb{R}_{2, b}^{+}:=\left[\mathbb{R}_{t}^{+} \times \mathbb{R}_{s}^{+} ;\{0\} \times\{0\}\right] .
$$

Then the acc triple heat space is constructed from $\mathcal{T} \times \mathbb{R}_{2, b}^{+}$by blowing up along 12 submanifolds, creating the following 12 boundary faces. Below, let $t D$ be the lift to $\mathcal{T}$ of the diagonal in $\mathcal{S} \times \mathcal{S}^{\prime} \times \mathcal{S}^{\prime \prime}$, let $D_{110}$ be the lift of the diagonal in $\mathcal{S} \times \mathcal{S}^{\prime}, D_{011}$ be the lift of the diagonal in $\mathcal{S}^{\prime} \times \mathcal{S}^{\prime \prime}$, and $D_{101}$ be the lift of the diagonal in $\mathcal{S} \times \mathcal{S}^{\prime \prime}$.

\begin{tabular}{ll}
\hline Submanifold blown up & Face created \\
\hline$Y \times Y^{\prime} \times Y^{\prime \prime} \times\{0\} \times\{0\}, d t, d s$ & $S_{11122}$ \\
$Y \times Y^{\prime} \times\{t=0\}, d t$ & $S_{11020}$, \\
$Y^{\prime} \times Y^{\prime \prime} \times\{s=0\}, d s$ & $S_{01102}$ \\
$Y \times Y^{\prime \prime} \times\{t=s=0\}, d s, d t$ & $S_{10122}$ \\
$Y \times Y^{\prime} \times Y^{\prime \prime}$ & $S_{111}$ \\
$Y \times Y^{\prime}$ & $S_{110}$ \\
$Y^{\prime} \times Y^{\prime \prime}$ & $S_{011}$ \\
$Y \times Y^{\prime \prime}$ & $S_{101}$ \\
$t D \times\{t, s=0\}, d s, d t$ & $S_{t d}$ \\
$D_{110} \times\{t=0\}, d t$ & $S_{d 20}$ \\
$D_{011} \times\{s=0\}, d s$ & $S_{d 02}$ \\
$D_{101} \times\{s=t=0\}, d s, d t$ & $S_{d 22}$ \\
\hline
\end{tabular}

As constructed, the acc triple heat space has full and partial projection/ blow-down maps to three identical copies of the acc heat space, the left, right and center, and to three corresponding copies of the blown-down space $\left\{\epsilon=\epsilon^{\prime}\right\} \subset \mathcal{S}^{2} \times \mathbb{R}^{+}$. To compose two elements $A$ and $B$, we view the element $A$ as acting from the left to the right while $B$ acts from the right to the center. Formally, the composition $B \circ A$ is the push forward from the acc triple heat 
space of the product of the lifts of $A$ and $B$. Compatibility assumptions on the leading orders of $A$ and $B$ at boundary faces of the acc heat space are required so that we can push forward. With these assumptions and with the possible inclusion of normalizing factors at boundary faces of the acc heat space, two elements compose as one would expect. The technical details in the proof of this composition rule are expected to be analogous to the technical details in the proof of the ac scattering heat calculus composition rule (Appendix A). Although the full acc triple heat space and composition rule are not necessary in our analysis of the heat kernels, its construction may be an instructive model for employing our methods and those of [33] to construct solution kernels of geometric operators under conic and more general metric degenerations.

\section{References}

[1] P. Albin, The Gauss-Bonnet theorem and index theory on conformally compact manifolds, $\mathrm{PhD}$ dissertation, Stanford University, June 2005.

[2] J. Brüning, J. Gil, D. Grieser and M. Lesch, The resolvent expansion on singular spaces, Advances in Partial Differential Equations, Approaches to Singular Analysis, Birkhauser, Boston, 2001, 208-233.

[3] J. Brüning and R.T. Seeley, The expansion of the resolvent near a singular stratum of conical type, J. Funct. Anal. 95 (1991), 255-290.

[4] I. Chavel, Eigenvalues in Riemannian Geometry, Academic Press, 1984.

[5] J. Cheeger, On the spectral geometry of spaces with cone-like singularities, Proc. Natl. Acad. Sci. USA 76 (1979), 2103-2106.

[6] - Spectral geometry of singular Riemannian spaces, J. Diff. Geom. 18 (1984), 575-657.

[7] J. Cheeger and T. Colding, On the structure of spaces with Ricci curvature bounded below, I, J. Diff. Geom. 46(3) (1997), 406-480.

[8] - On the structure of spaces with Ricci curvature bounded below, II, J. Diff. Geom. 54(1) (2000), 13-36.

[9] - On the structure of spaces with Ricci curvature bounded below, III, J. Diff. Geom. 54(1) (2000), 37-74.

[10] A. Degeratu and R. Mazzeo, Fredholm results on QALE manifolds, in preparation. 
[11] Y. Ding, Heat kernels and Green's functions on limit spaces, Commun. Anal. Geom. 10(3) (2002), 475-514.

[12] C. Epstein, R. Melrose and G. Mendoza, The Heisenberg algebra, index theory and homology, http://math.mit.edu/ rbm/book.html, in preparation.

[13] K. Fukaya, Collapsing of Riemannian manifolds and eigenvalues of Laplace operator, Invent. Math. 87(3) (1987), 517-547.

[14] M. Gaffney, A special Stokes' theorem for complete Riemannian manifolds, Ann. Math. 60 (1954), 140-145.

[15] J. Gil, Full asymptotic expansion of the heat trace for non-self-adjoint elliptic cone operators, Math. Nachr. 250 (2003), 25-57.

[16] J. Gil and G. Mendoza, Adjoints of elliptic cone operators, Amer. J. Math. 125(2) (2003), 357-408.

[17] A. Hassell and A. Vasy, The resolvent for Laplace-type operators on asymptotically conic spaces, Ann. l'Inst. Fourier 51 (2001), 1299-1346.

[18] A. Hassell, R. Mazzeo and R. Melrose, A signature formula for manifolds with corners of codimension 2, Topology 36(5) (1997), 1055-1075.

[19] L. Hörmander, The spectral function of an elliptic operator, Acta. Math. 121 (1968), 193-218.

[20] - The analysis of linear partial differential operators, Vol. 1, Springer-Verlag, 1987.

[21] D. Joyce, Compact Manifolds with Special Holonomy, Oxford University Press, 2000.

[22] S. Krantz, Partial Differential Equations and Complex Analysis, CRC Press, 1992.

[23] N. Lebedev, Special Functions and Their Applications, Prentice-Hall, Inc., 1965.

[24] M. Lesch, Differential operators of Fuchs type, conical singularities, and asymptotic methods, Teubner Texte zur Mathematik, Vol. 136, Teubner-Verlag, Leipzig, 1997.

[25] J. Lott, Remark about the spectrum of the p-form Laplacian under a collapse with curvature bounded below, Proc. AMS 132 (2003), 11-918. 
[26] - Collapsing and the differential form Laplacian: the case of a singular limit space, http://www.math.lsa.umich.edu/ lott, in preparation.

[27] P. Loya, Asymptotic properties of the heat kernel on conic manifolds, Israel J. Math. 136 (2003), 285-306.

[28] R. Mazzeo, Elliptic theory of differential edge operators I, Comm. Partial Diff. Eqn. 16(10) (1991), 1615-1664.

$[29] \longrightarrow$, Resolution blowups, spectral convergence and quasiasymptotically conic spaces, Journées équations aux dérivées partielles, 2006, Art. no. 8, 16p.

[30] R. Mazzeo and R. Melrose, Analytic surgery and the eta invariant, Geom. Funct. Anal. 5(1), (1995), 14-75.

[31] R. Mazzeo and J. Rowlett, A heat invariant anomaly on polygonal domains, in preparation.

[32] P.T. McDonald, The Laplacian for spaces with cone-like singularities, Ph.D. Dissertation, Massachusetts Institute of Technology, 1990.

[33] R. Melrose, The Atiyah-Patodi-Singer Index Theorem, Research Notes in Mathematics, 4, A K Peters, Ltd, 1993.

[34] E. Mooers, Heat kernel asymptotics on manifolds with conic singularities, J. Anal. Math. 78 (1999), 1-36.

[35] W. Müller, On the index of Dirac operators on manifolds with corners, J. Diff. Geom. 49 (1996), 97-177.

[36] S. Rosenberg, The Laplacian on a Riemannian Manifold, Cambridge University Press, 1997.

[37] J. Rowlett, Spectral geometry and asymptotically conic convergence, $\mathrm{PhD}$ dissertation, Stanford University, June 2006.

[38] M.A. Shubin, Pseudodifferential Operators and Spectral Theory, Springer, 2000.

Department of Mathematics

South Hall RoOM 6607

UNIVERSITY OF CALIFORNIA

SANTA BARBARA, CA 93106

USA

E-mail address: rowlett@math.ucsb.edu

Received December 15, 2006 